\title{
Rod Outer Segment Development Influences AAV-Mediated Photoreceptor Transduction After Subretinal Injection
}

\author{
Lolita Petit, ${ }^{1}$ Shan Ma, Shun-Yun Cheng, ${ }^{1}$ Guangping Gao, and Claudio Punzo ${ }^{1,2, *}$ \\ ${ }^{1}$ Department of Ophthalmology and Gene Therapy Center, ${ }^{2}$ Department of Neurobiology, and ${ }^{3}$ Department of Microbiology and Physiological Systems \\ and Gene Therapy Center, University of Massachusetts Medical School, Worcester, Massachusetts.
}

Vectors based on the adeno-associated virus (AAV) are currently the preferred tools for delivering genes to photoreceptors (PR) in small and large animals. AAVs have been applied successfully in various models of PR dystrophies. However, unknown barriers still limit AAV's efficient application in several forms of severe PR degenerations due to insufficient transgene expression and/or treated cells at the time of injection. Optimizations of PR gene therapy strategies will likely benefit from the identification of the cellular factors that influence PR transduction. Interestingly, recent studies have shown that the AAV transduction profile of PRs differs significantly between neonatal and adult mouse retinas after subretinal injection. This phenomenon may provide clues to identify host factors that influence the efficiency of AAV-mediated PR transduction. This study demonstrates that rod outer segments are critical modulators of efficient AAV-mediated rod transduction. During retinal development, rod transduction correlated temporally and spatially with the differentiation order of PRs when vectors were introduced subretinally but not when introduced intravitreally. All subretinally injected vectors had an initial preference to transduce cones in the absence of formed rod outer segments and then displayed a preference for rods as the cells matured, independently of the expression cassette or AAV serotype. Consistent with this observation, altered development of rod outer segments was associated with a strong reduction of rod transduction and an increase in the percentage of transduced cones by 2to 2.8 -fold. A similar increase of cone transduction was observed in the adult retinal degeneration 1 (rd1) retina compared to wild-type mice. These results suggest that the loss of rod outer segments in diseased retinas could markedly affect gene transfer efficiency of AAV vectors by limiting the ability of AAVs to infect dying rods efficiently. This information could be exploited for the development of more efficient AAV-based PR gene delivery procedures.

Keywords: AAV, retina, photoreceptors, cones, rods, gene therapy

\section{INTRODUCTION}

RECOMBINANT AAV SEROTYPE 2 (AAV2) is currently being evaluated in Phase 1/2 and 3 gene therapy clinical trials for treating diverse inherited retinal diseases involving the retinal pigment epithelium (RPE), such as Leber congenital Amaurosis $2{ }^{1-9}$ retinitis pigmentosa, ${ }^{10}$ and choroideremia. ${ }^{11,12}$ These studies demonstrate that subretinal delivery of AAV is safe and can provide rescue of vision-guided be- havior in patients with advanced disease at least for several years. ${ }^{13}$ However, broader application of retinal gene therapy will require efficient transduction of other retinal cells, in particular photoreceptors (PR).

The ability of various AAV serotypes to transduce PRs has been evaluated in small ${ }^{14-18}$ and large ${ }^{15,19-26}$ animals. Among these, pseudotypes $-2 / 5,-2 / 7,-2 / 8,-2 / 9$, and $-2 / \mathrm{rh} 10$ were found to have the highest transduction efficiencies for PRs after

${ }^{*}$ Correspondence: Dr. Claudio Punzo, University of Massachusetts Medical School, 368 Plantation Street, Albert Sherman Center, AS6-2041, Worcester, MA 01605-2324 E-mail: Claudio.Punzo@umassmed.edu

(c) Lolita Petit et al. 2017; Published by Mary Ann Liebert, Inc. This is an Open Access article distributed under the terms of the Creative Commons Attribution License, which permits unrestricted use, distribution, and reproduction in any medium, provided the original work is properly cited. 
subretinal delivery. ${ }^{27,28}$ These vectors have been used to drive persistent transgene expression successfully, resulting in improvements of the retinal structure and/or function in a number of animal models of PR dystrophies, in particular in models of slow PR death. ${ }^{13}$ Yet, in models of severe PR degeneration, many $\mathrm{PR}$ therapies have not been very effective at rescuing PR over the long term. This is likely due to the difficulties in treating sufficient cells and/or expressing therapeutic levels of the transgene in a timely manner. In support of this idea, AAV8-based vectors have provided higher benefits than AAV5 in different disease models of primary PR dystrophies, such as in the retinal degeneration 10, the Rpgrip $1^{-/-}$(retinitis pigmentosa GTPase regulator interacting protein 1 ), the

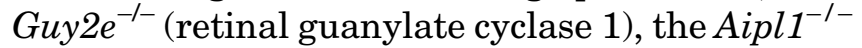
(aryl hydrocarbon interacting protein like 1), and the $R \mathrm{ho}^{-l-}$ (rhodopsin) mice, consistent with the superior ability of AAV8 to transduce PRs more efficiently and thus delivering higher transgene levels when compared to AAV5 (for review, see Petit et $a l .{ }^{13}$ ). Moreover, a recent study has shown that a therapeutic transgene can halt degeneration, regardless of the severity of PR loss if activated optimally in all PR cells. ${ }^{29}$ These results point to the importance of improving PR transduction efficiency to maximize clinical impact, and indicate that further development of retinal gene therapy will likely benefit from a better understanding of the potential host cell factors that restrict $\mathrm{PR}$ transduction in vivo. However, basic aspects of AAV-PR interactions are still unknown, and mechanisms affecting PR transduction after subretinal injection remain largely unexplored. ${ }^{20}$

Interestingly, recent reports have shown that after subretinal injection, the AAV transduction profile of $\mathrm{PR}$ cells changes dramatically between neonatal and adult mice. ${ }^{30-34}$ In particular, injection of AAV2/8 at postnatal day (PND) 0 results primarily in cone transduction ( $\sim 100 \%$ of cones, almost no rods), ${ }^{32-34}$ whereas the vast majority of transduced cells in adults are rods. ${ }^{14,15,32}$ The current study took advantage of this shift in tropism to identify host factors critical for AAVmediated PR transduction. Using 13 different AAV serotypes, cone and rod transduction were specifically assessed after AAV subretinal delivery in the developing retina and in two murine models of rodcone dystrophies that display either an arrest of PR outer segment development or a rapid loss of rod cells. Irrespective of the serotype tested, it was found that all vectors preferentially transduced cones when delivered at PND1, whereas delivery at PND21 resulted in a decrease in the percentage of transduced cones and a dramatic increase in the percentage of transduced rods. Additional injections at PND5 and PND10 showed that rod transduction temporally and spatially correlates with the development of their inner and outer segments. Furthermore, in mutant mouse models that lack rod outer segments or rod PR cells, a preferential transduction of cones was observed. Together, these findings indicate that the access of AAV vectors to PR cells through their segments is a key factor for PR transduction upon subretinal delivery. Additionally, the data suggest that mature rods and cones may compete for AAV access. These observations begin to define a new paradigm for AAV-PR interactions upon subretinal delivery, which should impact the development of more efficient AAV-based PR gene delivery procedures by providing an understanding of host factors required for successful AAV transduction.

\section{MATERIAL AND METHODS}

\section{Plasmid construction, AAV vector production, and purification}

Recombinant AAV2/5-CMV-GFPd, AAV2/5-CMVH2bGFP, AAV2/5-CMV-Cre, AAV2/5-pQCMV-H2b GFP, and AAV2/5-mCAR-H2bGFP were produced using the pAAV2-CMV-GFPd, pAAV2-CMV-H2bGFP, pAAV2-CMV-Cre, pAAV2-pQCMV-H2bGFP, or pAAV2-mCAR-H2bGFP plasmids, respectively. The pAAV2-CMV-GFPd plasmid expresses the destabilized GFP cDNA under the control of a human CMV enhancer/promoter, a human $\beta$-globin intron and an SV40 polyA signal, flanked by two AAV2 inverted terminal repeat sequences. The pAAV2-CMVH2bGFP plasmid expresses a histone 2B fused GFP (nuclear GFP). It was constructed by replacing the $G F P d$ cDNA with the H2bGFP sequence derived from the parental pQCMV-H2bGFP plasmid. The pAAV2-CMV-Cre plasmid expresses a Cre recombinase. It was constructed by replacing the $G F P d$ cDNA with the Cre sequence derived from the parental pQCMV-H2bGFP-I-Cre plasmid. The pAAV2-mCAR-H2bGFP plasmid was made by replacing the CMV promoter with the mouse cone arrestin (mCAR) promoter from the parental pQCMVH2bGFP plasmid. $^{35}$

scAAV1, -2, -3b, -4, -5, -6, -7, -8, -9, rh8, rh10, rh39, and rh43 expressing enhanced GFP (eGFP) under the control of the CB6 promoter were produced using the pAAVsc-CB6-eGFP plasmid, which bears a CB6 promoter, a rabbit globin polyA, and engineered ITRs for scAAV vector. The CB6 sequence was previously described $^{36}$ and includes a CMV enhancer/beta-actin (CB) promoter with a CMV IE enhancer. 
AAV2/5-CMV-GFPd, AAV2/5-CMV-H2bGFP, AAV2/5-CMV-Cre, AAV2/5-pQCMV-H2bGFP, and AAV2/5-mCAR-H2bGFP were produced in the Punzo laboratory by triple transfection of 293 cells according to previously reported methods ${ }^{37}$ and purified by two rounds of $\mathrm{CsCl}_{2}$ ultracentrifugation. scAAV vector production was carried out by the Vector Core of the Horae Gene Therapy Center of UMASS Medical School (Worcester, MA) ${ }^{38}$ Viral vector titers were determined simultaneously for all batches by quantitative real-time PCR (qPCR) with primers directed toward SV40pA (forward: 5' CGAGTGCTTTATTTGTGAAATTTG-3'; reverse: 5'-GGGGTTCCTTGTAGTTAATGA-3') or eGFP (forward: 5'-AGCAAAGACCCCAACGAGAA-3'; reverse: 5'-GGCGGCGGTCACGAA-3') and expressed as vector genome per milliliter $(\mathrm{vg} / \mathrm{mL})$. The final vector titers were between $8.1 \times 10^{12}$ and $5.1 \times 10^{13}$ $\mathrm{vg} / \mathrm{mL}$ (Table 1 ).

\section{Animals}

The CD1, Ai9 Cre reporter mice, and Pde6 $\beta^{r d 1 / r d 1}$ mice were purchased from the Jackson Laboratory. The M-opsin-Cre mice ${ }^{39}$ (cone-specific Cre line) and the rhodopsin knockout $\left(R h o^{-1-}\right)$ mice $^{40}$ were provided by Yun Z. Le (University of Oklahoma Health Sciences Center) and Janis Lem (Tufts University, Boston), respectively. $\mathrm{Ai}^{+/-} \mathrm{MCr}^{+}$ mice were generated by crossing $\mathrm{MCr}^{+}$mice with the Ai9 Cre reporter mice. Pde6 $\beta^{r d 1 /+}{ }_{-} \mathrm{Ai}^{+/-}$ mice were generated by crossing $r d 1$ mice and Ai9 mice. Heterozygotes were mated to produce Pde6 $\beta^{r d 1 / r d 1 /+} A_{-} i^{+/-}$mice.

All animals were maintained at UMASS Medical School under a 12 hour/12 hour light/dark cycle with unrestricted access to food and water. Lighting conditions were kept constant in all cages, with illumination ranging between 10 and 15 lux. All experiments involving mice were conducted in compliance with the Association for Research in Vision and Ophthalmology statement for the use of animals in ophthalmic and vision research. All procedures were approved by Institutional Animal Care and Use Committee of UMASS Medical School.

\section{Subretinal administration of rAAV vectors}

The same experimenter (L.P.) performed all subretinal injections of AAV vectors. Subretinal injection of AAV vectors was performed using a trans-scleral approach through the posterior part of the sclera, as previously described, ${ }^{41}$ with the following modifications. Injections were performed using thin-wall beveled glass micropipettes (Clunbury Scientific LLC) without sclerotomy. In mice older than PND1, a small hole was made at the transition of the cornea and sclera with a 33gauge needle before the injection in order to release intraocular pressure and allow for the formation of a vector bleb upon subretinal injection. This procedure allowed the vector bleb to occupy $40-60 \%$ of the retinal surface without injection-related damage. Fast green dye was added to the AAV preparations at a final concentration of $0.1 \%$ as a tracer to visualize the location of injection and thus ensure that AAV vectors were injected into the subretinal space. Mice received $0.5-0.75 \mu \mathrm{L}$ of vectors at PND1, 1-1.5 $\mu \mathrm{L}$ of vectors at PND5-10, and 1.5$2.5 \mu \mathrm{L}$ of vectors at PND21.

\section{Intravitreal administration of rAAV vectors}

Intravitreal injections were performed in PND1 mice, as previously described, ${ }^{41}$ using the same glass micropipettes (Clunbury Scientific LLC) introduced through the cornea-scleral margin. Fast green dye was added to AAV preparations at a final concentration of $0.1 \%$ as a tracer to visualize the location of injection and thus ensure that AAV vectors were injected into the intravitreal cavity. Mice received $0.75-1 \mu \mathrm{L}$ of vectors.

\section{Quantification of cone and rod transduction}

In all cases, the analysis was performed 3 weeks post AAV injection. The total number of retinas analyzed in each experimental group is indicated in Table 1. The efficiency of retinal transduction was first assessed under an inverted fluorescent microscope, and only retinas that displayed native GFP fluorescence in $>30 \%$ of the total retinal surface were processed for further analysis. All images were acquired with the Leica DM5500 fluorescent microscope equipped with a motorized stage for tiling and z-stack image acquisition and deconvolution software for confocal-like image quality.

Quantification of cone and rod transduction in selected retinas was performed using three independent methods. First, for each group of injected mice, a minimum of two retinas were entirely sectioned ( $20 \mu \mathrm{m} /$ section) and collected serially on five slides, such that each slide contained a representation of the entire eye. A minimum of two slides were processed for immunohistochemistry using Cy3-labeled peanut agglutinin lectin (PNA; 1:500; Vector Laboratories) and rabbit anti-cone arrestin (1:500; EMD Millipore). Images were taken from 510 sections at $40 \times$ over the entire transduced area at different focal plans ( $\mathrm{z}$ stacks) using epifluorescence microscopy (Supplementary Fig. S1; Supplementary Data are available online at www.liebertpub.com/hum). The acquired epifluorescent images were then deconvolved in order to 
obtain confocal-like resolution by eliminating the out-of-focus signal that could be misinterpreted as colocalization (Supplementary Fig. S1a). Cone quantification was performed in one selected $\mathrm{z}$ stack for each image, based on the colocalization of the GFP signal with the cone arrestin marker (usually at the level of cell nuclei). Rod quantification was performed within the same $z$ stack for each image by manually counting the number of total $\mathrm{GFP}^{+} \mathrm{PRs}$ and the number of $\mathrm{GFP}^{+}$cones per image, and by determining through an automated counting algorithm the total number of PR nuclei in the outer nuclear layer (ONL) of the same image using the Imaris software package (Supplementary Fig. S1b). Rod transduction was then calculated as an average per eye and per group by determining the ratio of the number of total $\mathrm{GFP}^{+}$ $\mathrm{PRs}$ - number of $\mathrm{GFP}^{+}$cones to the total number of PR nuclei - number of cone cells per image.

Second, for groups of mice injected with AAV$d G F P$ or AAV-eGFP vectors, cone quantification was also performed on retinal flat mounts stained with the following lectins/antibodies: Cy3-labeled PNA (1:500; Vector Laboratories), rabbit anti-cone arrestin (1:500; EMD Millipore; AB15282), rabbit anti-LM opsin (1:500; EMD Millipore; AB5405), and goat anti-S opsin (1:500; Santa Cruz Biotechnology; SC-14363). For each flat mount, four to six representative $100 \mu \mathrm{m}^{2}$ areas within the transduced area (center and periphery) were analyzed. The percentage of transduced cones was determined as an average per eye and per group by quantifying the number of $\mathrm{GFP}^{+}$cones over the total number of cones in the same areas. This quantification was performed at the level of cone segments, where GFP fluorescence in cones was unambiguously surrounded by PNA staining (Supplementary Fig. S1c).

Third, AAV5-CMV-H2bGFP and AAV5-pQCMV$H 2 b G F P$ vectors were also evaluated in $\mathrm{Ai}^{+}{ }_{-} \mathrm{MCre}$ mice, which express CRE-mediated td-Tomato (red) in the entire cone cell bodies (Supplementary Fig. S1d).

\section{Cell lines}

Two different Chinese hamster ovary (CHO) cell lines were used to confirm the specificity of the lectins used in this study. These include the parental cell line Pro5 and sialic-acid deficient cell line Lec2. Both cell lines were kindly provided by Miguel Sena-Esteves (UMASS Medical School). Cells were cultured in Dulbecco's modified Eagle's medium (DMEM) supplemented with $10 \%$ fetal bovine serum and 1\% streptomycin and maintained at $37^{\circ} \mathrm{C}$ with $5 \% \mathrm{CO}_{2}$. Cells were seeded at $10^{5}$ cells/well in 12-well plates prior to treatment for $1 \mathrm{~h}$ at $37^{\circ} \mathrm{C}$ with DMEM alone or DMEM supplemented with $50 \mathrm{mIU} / \mathrm{mL}$ of neuraminidase type III from Vibrio cholerae (Sigma-Aldrich; N7785). Cells were then washed three times with DMEM, once with PBS- $1 \times$ and fixed with $4 \%$ PFA for $10 \mathrm{~min}$. Cells were subjected to staining for $1 \mathrm{~h}$ in PBS- $1 \times$ at room temperature $(\mathrm{RT})$ using the following lectins: Cy2- or Cy3-labeled PNA (1:500), Cy2-conjugated Maackia amurensis lectin (MAL1; 1:500), biotinylated Erythrina cristagalli lectin (ELC; 1:500), and Cy5-conjugated wheat germ agglutinin (WGA; 1:500) all from Vector Laboratories. After three washes with ice-cold PBS- $1 \times$ to remove unbound lectins, ECL was visualized using Streptavidin-Cy5 (Molecular Probes). Cells were imaged using an inverted fluorescence microscope.

\section{Lectin analysis}

\section{of the inter-photoreceptor matrix}

Analysis of the inter-photoreceptor matrix (IPM) was performed on retinal sections, flat mounts, and explants from Ai9_MCre ${ }^{+}$mice euthanized at PND1, PND5, PND10, PND14, and PND21, and on retinal flat mounts from $R h o^{-/-}$mice euthanized at PND21. Three eyes were used for each experimental group. Retinal sections and retinal flat mounts were processed and stained, as previously described, for the cell culture experiments, with the following panel of lectins in PBS1X: PNA (1:500; Vector Laboratories), MAL1 (1:500; Vector Laboratories), ELC (1:500; Vector Laboratories), and WGA (1:500; Vector Laboratories). For retinal explants treated with neuraminidase, retinas were dissected in cold DMEM and incubated in either DMEM alone or DMEM with $50 \mathrm{mIU} / \mathrm{mL}$ of neuraminidase type III from $V$. cholerae (SigmaAldrich; N7785) for $1 \mathrm{~h}$ at $37^{\circ} \mathrm{C}$. Retinal explants were then washed three times with cold DMEM, once with cold PBS-1×, and fixed in 4\% PFA for $30 \mathrm{~min}$. Retinal explants were subjected to lectin staining for $1 \mathrm{~h}$ in PBS-1× at RT. After three washes with ice-cold PBS- $1 \times$ to remove unbound lectins, retinal explants were flat mounted and analyzed by fluorescence microscopy (Leica DM5500).

\section{AAV-binding assay and qPCR}

AAV-binding assay was performed on three biological samples and repeated two to four times with retinal explants from PND1 and PND21 CD1 mice. Retinas were dissected in cold DMEM and incubated in either DMEM with or without neuraminidase for $1 \mathrm{~h}$ at $37^{\circ} \mathrm{C}$. Retinal explants were then washed three times with cold DMEM, and pre-chilled at $4^{\circ} \mathrm{C}$ for $1 \mathrm{~h}$ in $500 \mu \mathrm{L}$ of DMEM. AAV vectors were added at $1 \times 10^{10} \mathrm{vg} / \mathrm{well}$, and explants 
were incubated at $4^{\circ} \mathrm{C}$ for $1 \mathrm{~h}$. Retinal explants were then washed three times with cold DMEM and once with cold PBS- $1 \times$ to remove unbound AAV particles. Total DNA was extracted using the DNeasy Blood and Tissue Kit (Qiagen). AAV genome copies were quantified by real-time PCR with the aforementioned primers for the SV40pA or eGFP sequence. AAV vector genomes (vg) were normalized to the number of retinal cells, using primers complementary to the mouse beta actin DNA (forward: 5'-ACTGGGACGACATGGAGAAG3'; reverse: 5'-GGGGTGTTGAAGGTCTCAAA-3'). Samples were run in triplicate. The results were expressed as mean AAV vg per genome DNA.

\section{Localization of AAV binding to retinal photoreceptors}

Localization of AAV particles was assessed after subretinal injection of PND1 and PND21 Ai9_MCre ${ }^{+}$mice with scAAV8 or scAAV9 vectors. Eyes injected with PBS-1× were used as negative controls. One hour post injection, animals were euthanized and retinas processed for flat mount, as previously described. ${ }^{41}$ Retinas were incubated overnight at $4^{\circ} \mathrm{C}$ with polyclonal rabbit antibodies raised against intact capsids of AAV8 or AAV9 at 1:500 in blocking solution (kindly provided by G.G.). Sections were then incubated with a Cy2-conjugated secondary anti-rabbit antibody (Jackson ImmunoResearch; 1:500) for $2 \mathrm{~h}$ at RT. Results were analyzed by fluorescence microscopy (Leica DM5500).

\section{Statistical analysis}

All data are expressed as mean \pm standard deviation $(S D)$. All statistical comparisons use Student's $t$-test, with $p<0.05$ considered as statistically significant in all comparisons.

\section{RESULTS}

\section{Subretinal injection of AAV5 at PND1 predominantly targets cone photoreceptors}

Recent reports showed that subretinal injection of AAV8 into PND0 mice results primarily in cone transduction, whereas the vast majority of transduced cells after subretinal injection in adult mice are rods. ${ }^{32-34}$ To demonstrate further that PR postnatal development impacts the pattern of $P R$ transgene expression, neonatal mice (PND1) were subretinally injected with $6 \times 10^{8}-1.2 \times 10^{9} \mathrm{vg}$ of single-stranded (ss) AAV2/5-CMV-dGFP (Table 1 and Supplementary Fig. S2a). AAV5 was chosen because this serotype (i) has been shown to significantly target PR cells in the adult mouse, ${ }^{14,15,18}$ cat, ${ }^{26} \mathrm{dog},{ }^{23,42,43} \mathrm{pig},{ }^{15,22}$ and nonhuman prima- $\mathrm{te}^{21}$; (ii) has been used successfully to restore retinal function and preserve retinal structure in various models of $P R$ diseases ${ }^{13}$; and (iii) is currently planned in upcoming Phase I-II clinical trials for the treatment of PR diseases. ${ }^{13}$ Mice with successful subretinal injections were euthanized 3 weeks post injection (wpi), at PND21, after PR differentiation and maturation is completed (Fig. 1 and Supplementary Fig. S2). As previously described, ${ }^{30,33,34}$ subretinal injections at PND1 yielded GFP expression throughout the entire retinal surface (Fig. 1a and Supplementary Fig. S2a), presumably because the interactions between the RPE and the PR outer segments that could limit the spread of the vector have not yet formed at PND1. Robust transduction of RPE was observed across the retina (Supplementary Fig. S2b). High levels of GFP were also detected in the ONL, which contains rod and cone PR nuclei (Fig. 1c and Supplementary Fig. S2b), and in the corresponding PR segments (Fig. 1a and c and Supplementary Fig. S2b). Immunostaining with cone-specific markers (PNA and cone arrestin) revealed that the majority of the $\mathrm{GFP}^{+}$ cells were also positive for the cone-specific markers (Fig. 1a and c and Supplementary Fig. S2b). Hence, as predicted, the transduction of PRs was essentially relegated to cones when considering the percentage of $\mathrm{GFP}^{+}$cells (cones: $69.1 \pm 7.4 \%$; rods: $2.7 \pm 3.1 \%$ ). L/ $\mathrm{M}$ and $\mathrm{S}$ cones were equally transduced (Supplementary Fig. S3).

To rule out an influence of factors such as inhibition of CMV promoter activity or dGFP steady state level on the final transgene expression pattern, PR transduction was analyzed in mice injected at PND1 with five additional AAV5 vectors. These vectors carry different transgenes and/or ubiquitous or PR-specific promoters: AAV5-CMVH2bGFP, AAV5-CMV-Cre, AAV5-mCAR-H2bGFP, AAV5-pQCMV-H2bGFP, as well as a selfcomplementary (sc) AAV5-CB6-eGFP (Table 1). Though some construction-dependency was noted, tropism was similar for all vectors examined (Fig. 1b and c and Supplementary Fig. S2b). Hence, the findings suggest that the absence of efficient rod transduction following subretinal administration at PND1 is not dependent on factors that alter vector genome expression, as well as second-strand synthesis in the transduced cells, but rather on differences in entry of AAV into cones and rods at the time of delivery. Consistent with that, AAV8 vectors carrying different promoters, such as AAVhRK1-GFP, AAV-mCAR-GFP, or AAV-CMV-GFP, have also been seen to drive transgene expression predominantly in cones after subretinal injection in PND0 neonate mice. ${ }^{33,34}$ Moreover, efficient trans- 
Table 1. Quantification of cone and rod transduction in mouse retinas 3 weeks after subretinal injections of different $A A V$ vectors at different ages

\begin{tabular}{|c|c|c|c|c|c|c|c|}
\hline Group & Vector & Titer (vg/mL) & Strain & Age & Retinas analyzed & $\%$ Cones $(\mathrm{M} \pm \mathrm{SD})$ & $\%$ Rods $(\mathrm{M} \pm \mathrm{SD})$ \\
\hline 1 & \multirow[t]{7}{*}{ AAV5-CMV-dGFP } & \multirow[t]{7}{*}{$1.2 \times 10^{13}$} & \multirow[t]{4}{*}{ CD1 } & PND1 & 13 & $69.1 \pm 7.4$ & $2.7 \pm 3.1$ \\
\hline 2 & & & & PND5 & 7 & $56.2 \pm 18.3$ & $6.0 \pm 1.2$ \\
\hline 3 & & & & PND10 & 6 & $48.3 \pm 6.6$ & $18.2 \pm 8.3$ \\
\hline 4 & & & & PND21 & 3 & $39.9 \pm 2.2$ & $32.2 \pm 17.4$ \\
\hline 5 & & & $r d 1$ & PND21 & 3 & $90.5 \pm 4.4$ & NA \\
\hline 6 & & & \multirow{2}{*}{$R_{h o o^{-1-}}$} & PND1 & 4 & $59.4 \pm 2.9$ & $0.09 \pm 0.008$ \\
\hline 7 & & & & PND21 & 2 & $87.2 \pm 7.2$ & $0.9 \pm 0.2$ \\
\hline 8 & \multirow[t]{8}{*}{ AAV5-CMV-H2bGFP } & \multirow[t]{8}{*}{$1.3 \times 10^{13}$} & \multirow[t]{4}{*}{ CD1 } & PND1 & 6 & $65.8 \pm 9.7$ & $3.2 \pm 2.8$ \\
\hline 9 & & & & PND5 & 3 & $29.2 \pm 1.3$ & $2.8 \pm 0.6$ \\
\hline 10 & & & & PND10 & 3 & $40.5 \pm 6.6$ & $7.4 \pm 2.2$ \\
\hline \multirow[t]{2}{*}{11} & & & & PND21 & 3 & $41.4 \pm 13.6$ & $21.3 \pm 10.9$ \\
\hline & & & Aig/+ & PND1 & 3 & $69.5 \pm 7.6$ & $7.3 \pm 1.2$ \\
\hline 12 & & & $r d 1$ & PND21 & 3 & $84.9 \pm 2.9$ & NA \\
\hline 13 & & & \multirow[t]{2}{*}{$R h o^{-/-}$} & PND1 & 2 & $60.3 \pm 5.8$ & $1.5 \pm 0.6$ \\
\hline 14 & & & & PND21 & 4 & $81.2 \pm 1.6$ & $4.8 \pm 1.7$ \\
\hline 15 & AAV5-CMV-H2bGFP & $5.1 \times 10^{13}$ & $\mathrm{CD} 1$ & PND1 & 2 & $70.3 \pm 5.2$ & $9.8 \pm 4.4$ \\
\hline \multirow[t]{2}{*}{16} & \multirow[t]{3}{*}{ AAV5-pQCMV-H2bGFP } & \multirow[t]{3}{*}{$1.3 \times 10^{13}$} & CD1 & PND1 & 6 & $67.2 \pm 4.7$ & $17.9 \pm 26.6$ \\
\hline & & & Aig/+ & PND1 & 2 & $66.9 \pm 4.7$ & $4.4 \pm 2.3$ \\
\hline 17 & & & $R h o^{--}$ & PND21 & 4 & $71.1 \pm 6.6$ & $1.2 \pm 0.3$ \\
\hline 18 & AAV5-mCAR-H2bGFP & $1.3 \times 10^{13}$ & CD1 & PND1 & 3 & $51.1 \pm 4.4$ & $1.0 \pm 0.4$ \\
\hline 19 & AAV5-CMV-Cre & $1.3 \times 10^{13}$ & Aig/+ & PND1 & 5 & $60.6 \pm 9.2$ & $4.8 \pm 1.6$ \\
\hline 20 & scAAV1-CB6-eGFP & $1.6 \times 10^{13}$ & CD1 & PND1 & 5 & $50.7 \pm 10.7$ & $8.9 \pm 6.7$ \\
\hline 21 & scAAV2-CB6-eGFP & $9.8 \times 10^{12}$ & $\mathrm{CD} 1$ & PND1 & 3 & $47.4 \pm 8.3$ & $3.1 \pm 1.4$ \\
\hline 22 & scAAV3b-CB6-eGFP & & $\mathrm{CD} 1$ & PND1 & 6 & $12.3 \pm 3.6$ & $4.3 \pm 2.3$ \\
\hline 23 & scAAV4-CB6-eGFP & $1.2 \times 10^{13}$ & CD1 & PND1 & 3 & 0 & 0 \\
\hline 24 & \multirow[t]{5}{*}{ scAAV5-CB6-eGFP } & \multirow[t]{5}{*}{$1.4 \times 10^{13}$} & \multirow[t]{4}{*}{ CD1 } & PND1 & 6 & $62.5 \pm 7.8$ & $7.2 \pm 5.0$ \\
\hline 25 & & & & PND5 & 3 & $31.5 \pm 9.3$ & $14.8 \pm 3.6$ \\
\hline 26 & & & & PND10 & 4 & $19.6 \pm 3.3$ & $19.6 \pm 3.3$ \\
\hline 27 & & & & PND21 & 3 & $23.7 \pm 5.7$ & $38.4 \pm 11.5$ \\
\hline 28 & & & $r d 1$ & PND21 & 2 & $87.2 \pm 6.2$ & NA \\
\hline 29 & scAAV6-CB6-eGFP & $9.8 \times 10^{12}$ & CD1 & PND1 & 3 & $50.6 \pm 24.9$ & $6.9 \pm 3.3$ \\
\hline 30 & \multirow[t]{2}{*}{ scAAV7-CB6-eGFP } & \multirow[t]{2}{*}{$1.4 \times 10^{13}$} & \multirow[t]{2}{*}{ CD1 } & PND1 & 6 & $55.1 \pm 15.3$ & $7.2 \pm 2.5$ \\
\hline 31 & & & & PND21 & 3 & $25.5 \pm 5.2$ & $34.9 \pm 9.9$ \\
\hline 32 & \multirow[t]{2}{*}{ scAAV8-CB6-eGFP } & \multirow[t]{2}{*}{$9.1 \times 10^{12}$} & \multirow[t]{2}{*}{ CD1 } & PND1 & 4 & $57.4 \pm 11.7$ & $5.1 \pm 2.0$ \\
\hline 33 & & & & PND21 & 8 & $23.3 \pm 9.7$ & $46.3 \pm 8.6$ \\
\hline 34 & \multirow[t]{3}{*}{ scAAV9-CB6-eGFP } & \multirow[t]{3}{*}{$1.0 \times 10^{13}$} & CD1 & PND1 & 5 & $58.1 \pm 13.6$ & $6.9 \pm 1.2$ \\
\hline 35 & & & & PND21 & 9 & $23.5 \pm 6.7$ & $50.3 \pm 5.4$ \\
\hline 36 & & & $r d 1$ & PND21 & 3 & $91.6 \pm 2.8$ & NA \\
\hline 37 & scAAVrh8-CB6-eGFP & $8.1 \times 10^{12}$ & CD1 & PND1 & 3 & $57.3 \pm 15.2$ & $6.1 \pm 3.3$ \\
\hline 38 & scAAVrh10-CB6-eGFP & $9.7 \times 10^{12}$ & $\mathrm{CD} 1$ & PND1 & 7 & $69.8 \pm 12.8$ & $6.5 \pm 2.3$ \\
\hline 39 & & & & PND21 & 5 & $25.3 \pm 12.4$ & $54.8 \pm 4.6$ \\
\hline 40 & scAAVrh39-CB6-eGFP & $1.0 \times 10^{13}$ & $\mathrm{CD} 1$ & PND1 & 5 & $61.2 \pm 12.9$ & $6.4 \pm 4.7$ \\
\hline 41 & scAAVrh43-CB6-eGFP & $8.1 \times 10^{12}$ & $\mathrm{CD} 1$ & PND1 & 6 & $39.8 \pm 9.9$ & $4.4 \pm 1.9$ \\
\hline
\end{tabular}

AAV, adeno-associated virus; PND, postnatal day; NA, not applicable.

fection of rods has been previously obtained after PND0 electroporation of pCMV-H2bGFP, ${ }^{41}$ a plasmid DNA that carries the same expression cassette as the AAV5-CMV-H2bGFP. Finally, a higher number of $\mathrm{GFP}^{+}$rods was observed after administration of higher doses of AAV5-CMV-H2bGFP $\left(9 \times 10^{9}\right.$ vg; Fig. $1 \mathrm{~d}$ and Table 1$)$ and AAV5-mCAR$H 2 b G F P\left(>1 \times 10^{10} \mathrm{vg}\right)^{41}$ at PND1, indicating that efficient transduction of immature rods is possible at this age but dependent on the quantity of the vector injected (Fig. 1d and Table 1).

\section{Rod development coincides with increased rod transduction after subretinal delivery}

In mice, cones start to undergo their terminal mitosis to begin their subsequent differentiation before rods. Most rods in mouse are born over an extended developmental time period (E12 to PND5) with the peak of rod production around birth and their differentiation into mature rods continuing well into the postnatal period. ${ }^{44}$ To examine further how the PR transduction pattern correlates with rod PR development, GFP expres- 
a

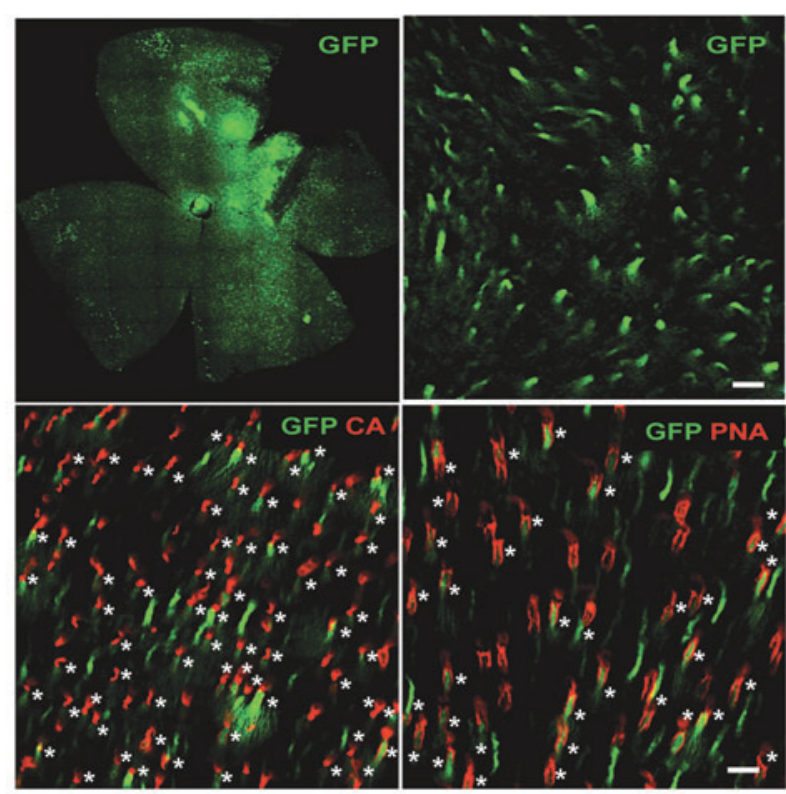

sSAAV5-CMV-dGFP

C

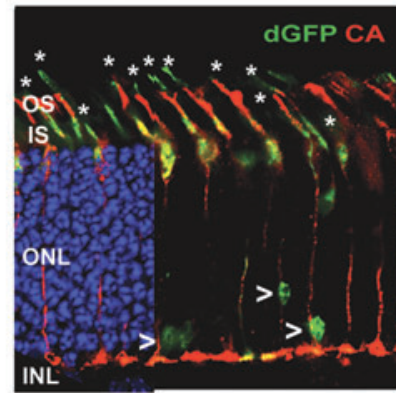

sSAAV5-CMV-dGFP

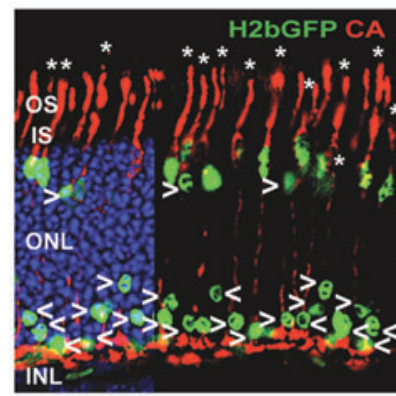

ssAAV5-pQCMV-H2bGFP

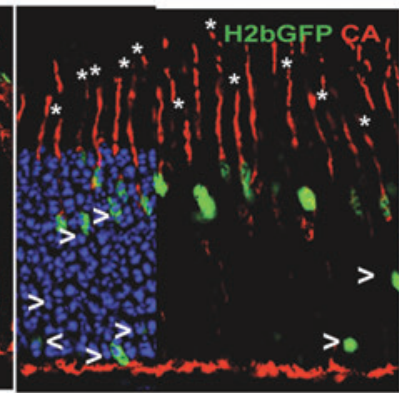

ssAAV5-CMV-H2bGFP

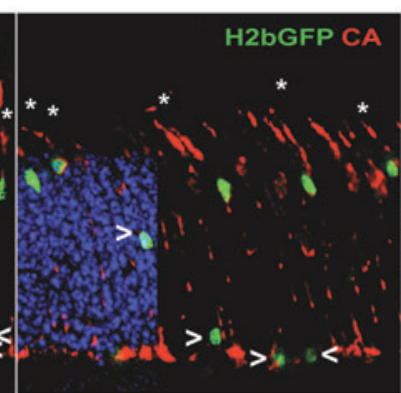

ssAAV5-mCAR-H2bGFP b

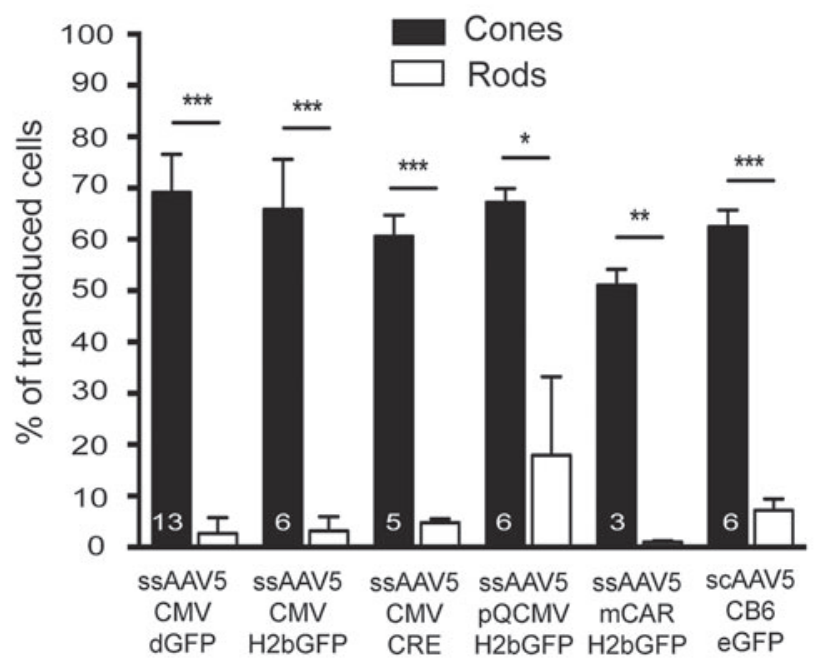

d

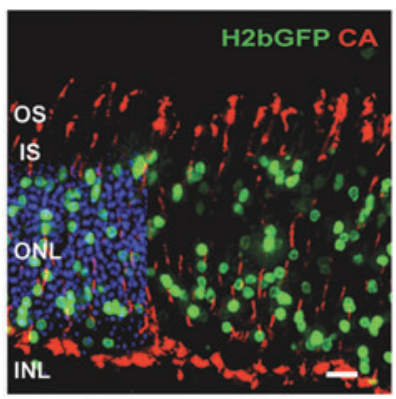

ssAAV5-CMV-H2bGFP

(high dose)

Figure 1. Photoreceptor transduction in the neonatal mouse retina by adeno-associated virus serotype 5 (AAV5) vectors. Mice injected on postnatal day 1 (PND1) were subretinally injected with similar doses of AAV2/5-CMV-dGFP, AAV2/5-CMV-H2bGFP, AAV2/5-CMV-CRE, AAV2/5-pQCMV-H2bGFP, AAV2/5-mCARH2bGFP, or scAAV2/5-CB6-eGFP vectors. (a) Representative retinal flat-mounts and (c, d) cryosections 3 weeks post injection (wpi) labeled with an antibody raised against anti-cone arrestin (CA) or with peanut agglutinin lectin (PNA; red) and counter stained with DAPI (blue: removed from $60 \%$ of panels to visualize red and green staining better) (c,d). AAV-mediated GFP was detected by its native fluorescence (green). AAV-mediated CRE expression was detected in Aig ${ }^{+}$ mice with the CRE-mediated expression of td-Tomato (white, native fluorescence). In retinal sections, retinal pigment epithelium (RPE) cells were artificially removed using Photoshop. Asterisks indicate $\mathrm{GFP}^{+}$or $\mathrm{CRE}^{+}$(td-Tomato ${ }^{+}$) cones, while arrowheads indicate $\mathrm{GFP}^{+}$or $\mathrm{CRE}^{+}$(td-Tomato ${ }^{+}$) rods, which are mainly found in the inner most rows of the outer nuclear layer (ONL). Quantitative analysis of the percentage of $\mathrm{GFP}^{+}$or $\mathrm{CRE}^{+}$photoreceptors in vector-exposed area is shown in (b). Error bars represent standard deviation (SD). Numbers in bars represent the number of retina analyzed. ${ }^{*} p<0.05$; ${ }^{* *} p<0.01$; ${ }^{* * *} p<0.001$ by Student's $t$-test. (d) After injection of higher dose of AAV2/5-pQCMV-H2bGFP, a higher number of GFP ${ }^{+}$rods is observed throughout the 0NL (representative section). Scale bars $=25 \mu \mathrm{m}$. INL, inner nuclear layer; IS, inner segments; OS, outer segments; sc, self-complementary; ss, single-strand. 
a
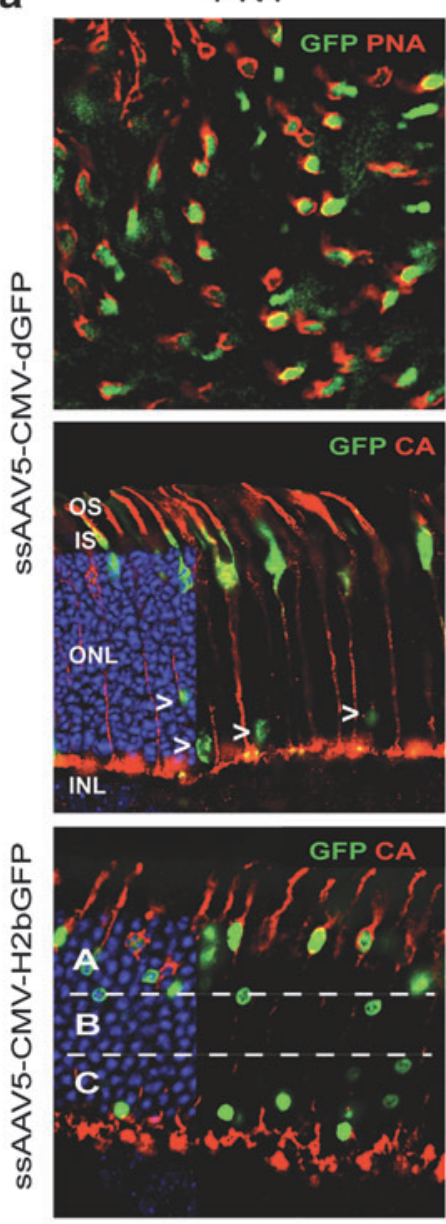

PN10
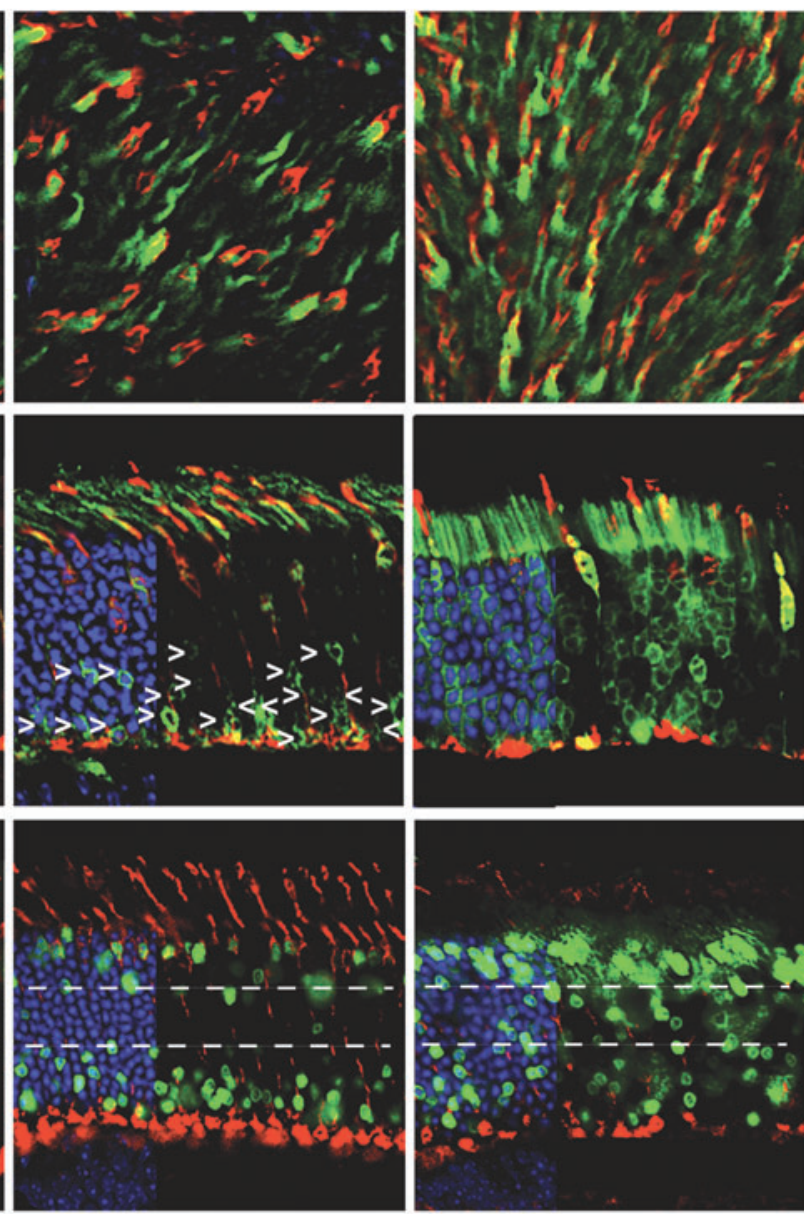

PN5
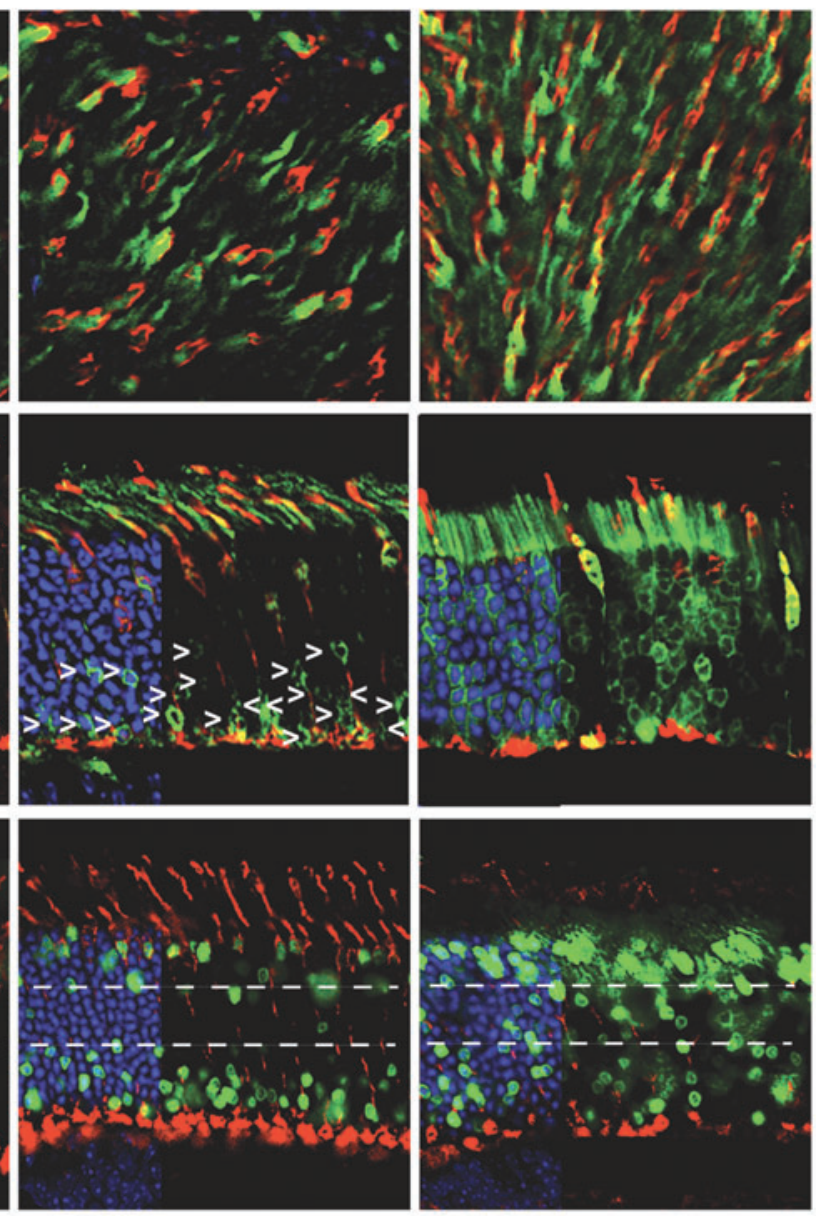

PN21
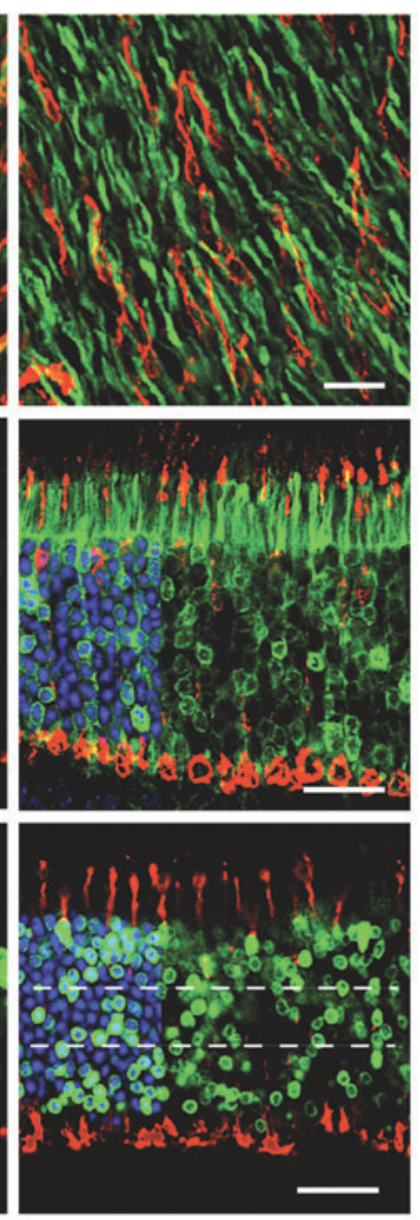
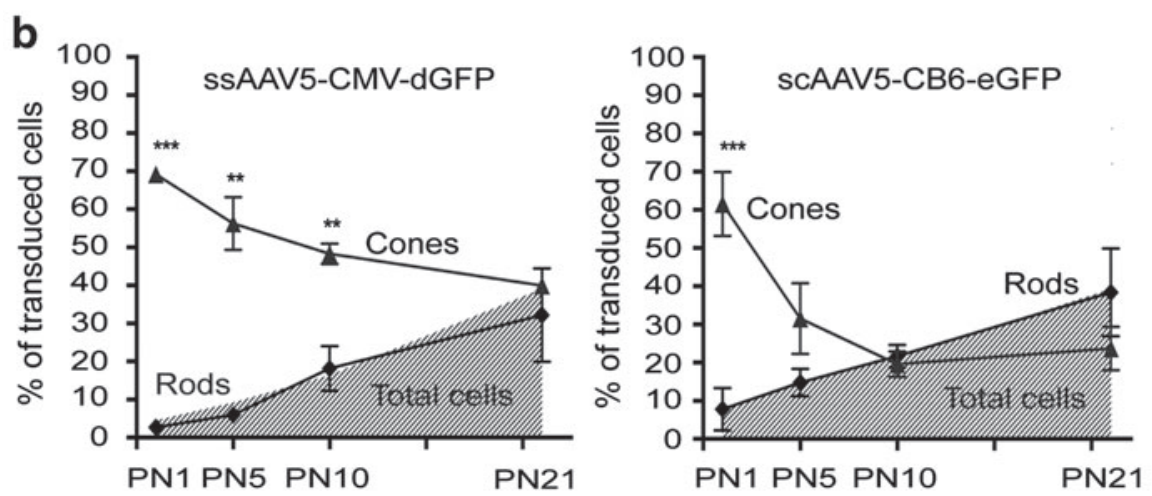

C

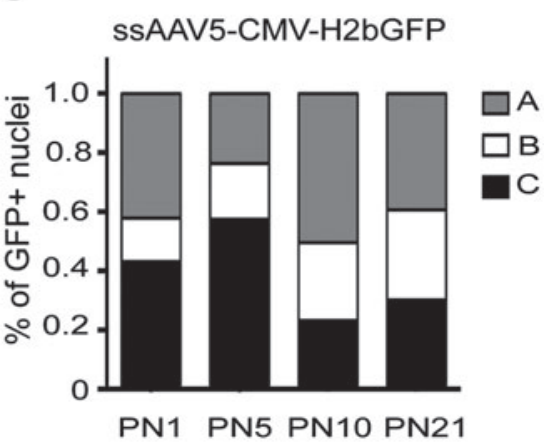

Figure 2. Efficiency of cone and rod photoreceptor transduction with AAV2/5 vectors at different times after birth. AAV2/5-CMV- $d G F P, A A V 2 / 5-C M V-H 2 b G F P$, and scAAV2/5-CB6-eGFP were subretinally injected in mice at PND1, PND5, PND10, and PND21. In all cases, analysis was performed 3 wpi. (a) Representative retinal flat mounts (upper row) and cryosections (middle row) from mice injected with AAV2/5-CMV-dGFP and cryosections (bottom row) from mice injected with AAV2/5-CMV-H2bGFP and immunostained for PNA or CA (red) and counterstained with DAPI (blue: removed from $60 \%$ of panels to visualize red and green staining better). AAV-mediated GFP was detected by its native fluorescence (green). RPE cells were artificially removed using Photoshop. Dotted horizontal lines divide the ONL in three sublayers showing that early injections (PND1 and PND5) result in the majority of transduced rods (arrowheads) located in the most inner part of the ONL. Arrowheads indicate $\mathrm{GFP}^{+}$rods (b). Quantification of cone, rod, and total photoreceptor transduction within the vector-exposed area after injection of AAV2/5-CMV-dGFP and AAV2/5-CB6-eGFP at the indicated ages. Results are shown as mean $\pm S D$. ${ }^{* *} p<0.01$ and ${ }^{* * *} p<0.001$ by Student's $t$-test. (c) Average distribution of $\mathrm{GFP}^{+}$nuclei within the ONL layer after AAV injection at the indicated ages. As shown in (a), the ONL was divided into three sublayers: $A$, outermost part of the $0 \mathrm{NL}$; $\mathrm{B}$, middle part of the $\mathrm{ONL}$; $\mathrm{C}$, innermost part of the $\mathrm{ONL}$. The percentage of $\mathrm{GFP}^{+}$nuclei/total nuclei $\left(\mathrm{DAPI}{ }^{+}\right.$) was calculated. Scale bars $=25 \mu \mathrm{m}$. 
sion was investigated in mice injected at three additional ages, corresponding to three different stages of PR development: PND5 (end of PR birth and early development of PR processes), PND10 (intermediate development of PR outer segments), and PND21 (PR development completed; Table 1). Injected retinas were all harvested at $3 \mathrm{wpi}$.

The histological analyses of three different AAV2/ 5 vectors (Fig. 2 and Supplementary Figs. S4 and S5) revealed a progressive shift in tropism from cones to rods that correlated with the differentiation of the retina. Indeed, after injection of AAV5-CMV-dGFP (Fig. 2a and b and Supplementary Fig. S4), AAV5CMV-H2bGFP (Fig. 2a), or scAAV5-CB6-eGFP (Supplementary Fig. S5a and Fig. 2b), the percentage of $\mathrm{GFP}^{+}$rods increased with the age of the mouse at the time of injection, whereas the percentage of $\mathrm{GFP}^{+}$cones decreased. As a result, injections of the three vectors at PND1 and PND5 directed GFP expression predominantly in cones, while a preferential transduction of rods was observed for injections at PND10 and PND21 (Table 1).

Interestingly, the location of $\mathrm{GFP}^{+}$rod nuclei in the ONL correlated with the timing of rod genesis (Fig. 2 and Supplementary Figs. S2b, S4, and S5a). After subretinal injection at PND1, the small percentage of transduced rod nuclei found was primarily located in the most inner row of the ONL where the first-born rods reside, as previously indicated by birth-dating experiments ${ }^{45-47}$ (Figs. 1 and $2 \mathrm{a}$ and c, and Supplementary Figs. S2b, S4, and S5a). After vector administration at PND5, while the number of transduced rods increased, $\mathrm{GFP}^{+}$ rods were still restricted in their location to the four or five most inner rows of the ONL (lower third of ONL; Fig. 2a and c and Supplementary Figs. S4 and S5a), consistent with the observation that newborn rods are stacked on the top of the earlier born ones. ${ }^{45}$ In contrast, injections after PND10 led to a wide distribution of $\mathrm{GFP}^{+}$nuclei across the ONL (Fig. 2a and c, and Supplementary Figs. S4 and S5a). In line with this finding, the transduction pattern of rod and cone PRs followed the central-toperipheral (data not shown) and ventral-to-dorsal gradient of $\mathrm{PR}$ differentiation ${ }^{48}$ when the vector was injected at PND3 (Supplementary Fig. S5b). Hence, the order of differentiation of PR cells is clearly correlated with the cells preferentially transduced after subretinal injection of AAV2/5.

\section{Photoreceptor development affects rod transduction, irrespective of AAV serotypes}

To determine whether PR development affects PR transduction by other AAV serotypes, neonatal mice were subretinally injected with a panel of
13 scAAV serotypes/variants, including AAV1, -2, $-3 b,-4,-5,-6,-7,-8,-9,-$ rh8, -rh10, -rh39, and -rh43, all expressing eGFP under the control of the CB6 promoter (Table 1, Figs. 3 and 4, and Supplementary Figs. S6 and S7). As controls, AAV5, -7, -8, -9, and -rh10 were subretinally injected into PND21 mice (Table 1 and Supplementary Fig. S6), as these vectors are known to exhibit excellent tropism for adult rods. ${ }^{14,15,18}$ Similar to the results observed after PND1 delivery of AAV5, PND1 injections of the all other AAVs resulted in preferential cone transduction when considering the percentage of $\mathrm{GFP}^{+}$cells, with the exception of AAV4, which efficiently transduced RPE cells only (Table 1 and Fig. 3). On average, AAVrh10 exhibited the most efficient transduction of cones (although not statistically different from AAV5, -6, -7, -8, -9, -rh8, and -rh39), whereas AAV3b-mediated transgene expression was limited to sporadic RPE and PRs (Fig. 3b). Notably, no significant difference in the percentage of transduced rods was seen between all the serotypes (Fig. 3). When considering the percentage of $\mathrm{GPF}^{+}$rods, all AAVs led to sparse rod transduction, with most of the $\mathrm{GFP}^{+}$rods localized in the innermost part of the ONL (Fig. 4 and Supplementary Figs. S6 and S7). Additionally, the preferential transduction of rods observed after subretinal injection of AAV2/5 vectors at PND21 was reproduced after injection of AAV7 (Supplementary Fig. S6d and e), -8 (Supplementary Fig. S6a, d, and e), -9 (Supplementary Fig. S6b, d, and e), and -rh10 (Supplementary Fig. S6c-e). Thus, rod PR development is a critical host factor that influences AAV tropism after subretinal delivery independently of the serotype and thus probably independently of the different cell attachments factors utilized by each capsid.

\section{Control of AAV transduction by photoreceptor development depends on the injection site}

Because a higher number of $\mathrm{GFP}^{+}$rods was observed after administration of higher doses of AAV5-CMV-H2bGFP $\left(9 \times 10^{9}\right.$ vg; Fig. $1 \mathrm{~d}$ and Table 1) and AAV5-mCAR-H2bGFP $\left(>1 \times 10^{10} \mathrm{vg}\right)^{41}$ at PND1, the overall absence of efficient rod transduction at PND1 may relate to the progressive loss of the episomal vector genomes in rods. AAV vectors integrate only at very low frequencies, and thus vector-mediated transgene expression is predicted to diminish over time under conditions of ongoing cellular proliferation. In transduced cones, the number of expressed vector genomes is likely to remain stable over time because cones are already post-mitotic by PND1-PND5. ${ }^{44}$ In contrast, although the vast majority of rod precursors are al- 
a

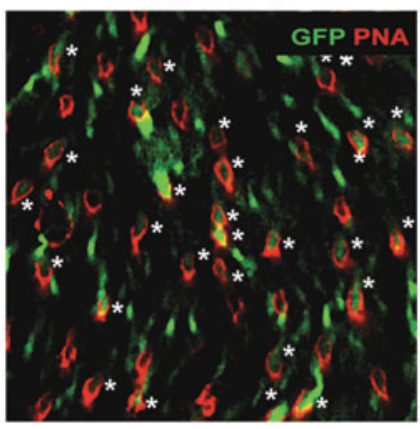

scAAV6

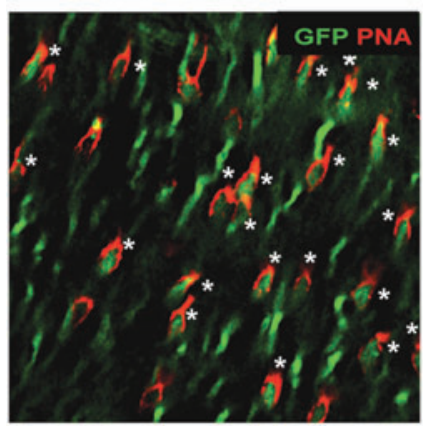

scAAVrh8

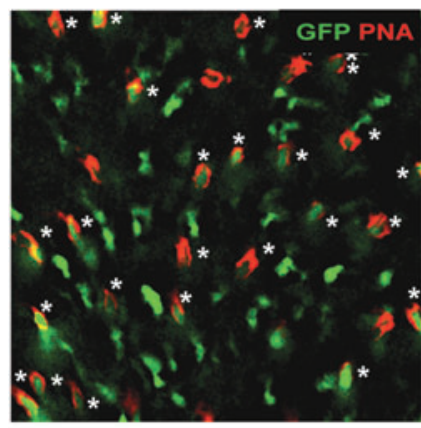

b

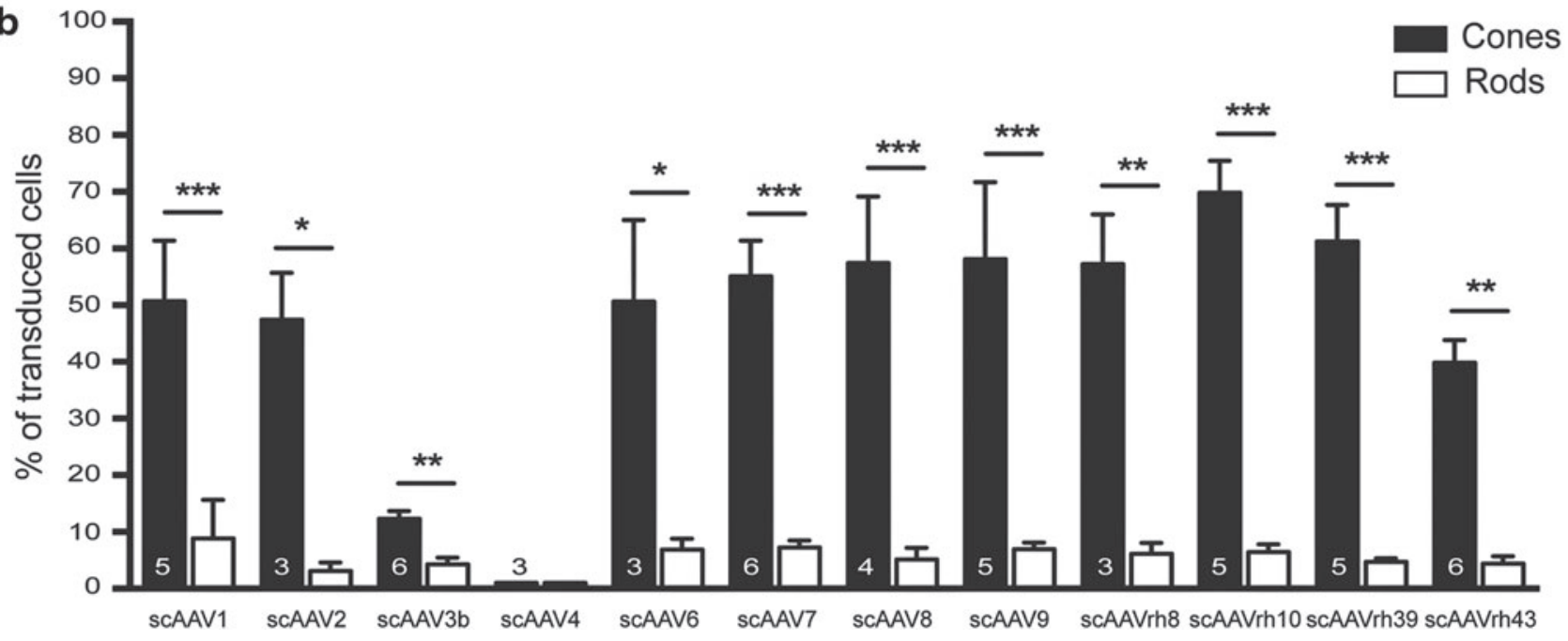

Figure 3. Predominant transduction of cone photoreceptors after the injection of 12 different AAV serotypes/variants at PND1. (a) Representative images of retinal flat mounts at 3 wpi of scAAV1, $-2,-3 b,-4,-6,-7,-8,-9$, -rh8, -rh10, -rh39, or -rh43 vectors (all expressing eGFP under the control of the CB6 promoter), as indicated. Labeling with PNA (red) shows expression of eGFP (green) predominantly in cones (asterisks), except for AAV4. (b) Quantification performed on retinal flat mounts and/or cryosections of the percentage of $\mathrm{GFP}^{+}$cones and $\mathrm{GFP}^{+}$rods in the vector exposed area (note: actual transduction efficiency of scAAV4 was $0 \%$ for rods and cones). Results are expressed as mean $\pm S D$. Numbers in bars represent the number of retinas analyzed. ${ }^{*} p<0.05 ;{ }^{* *} p<0.01$; ${ }^{* * *} p<0.001$ by Student's $t$-test. Scale bar $=25 \mu \mathrm{m}$. 


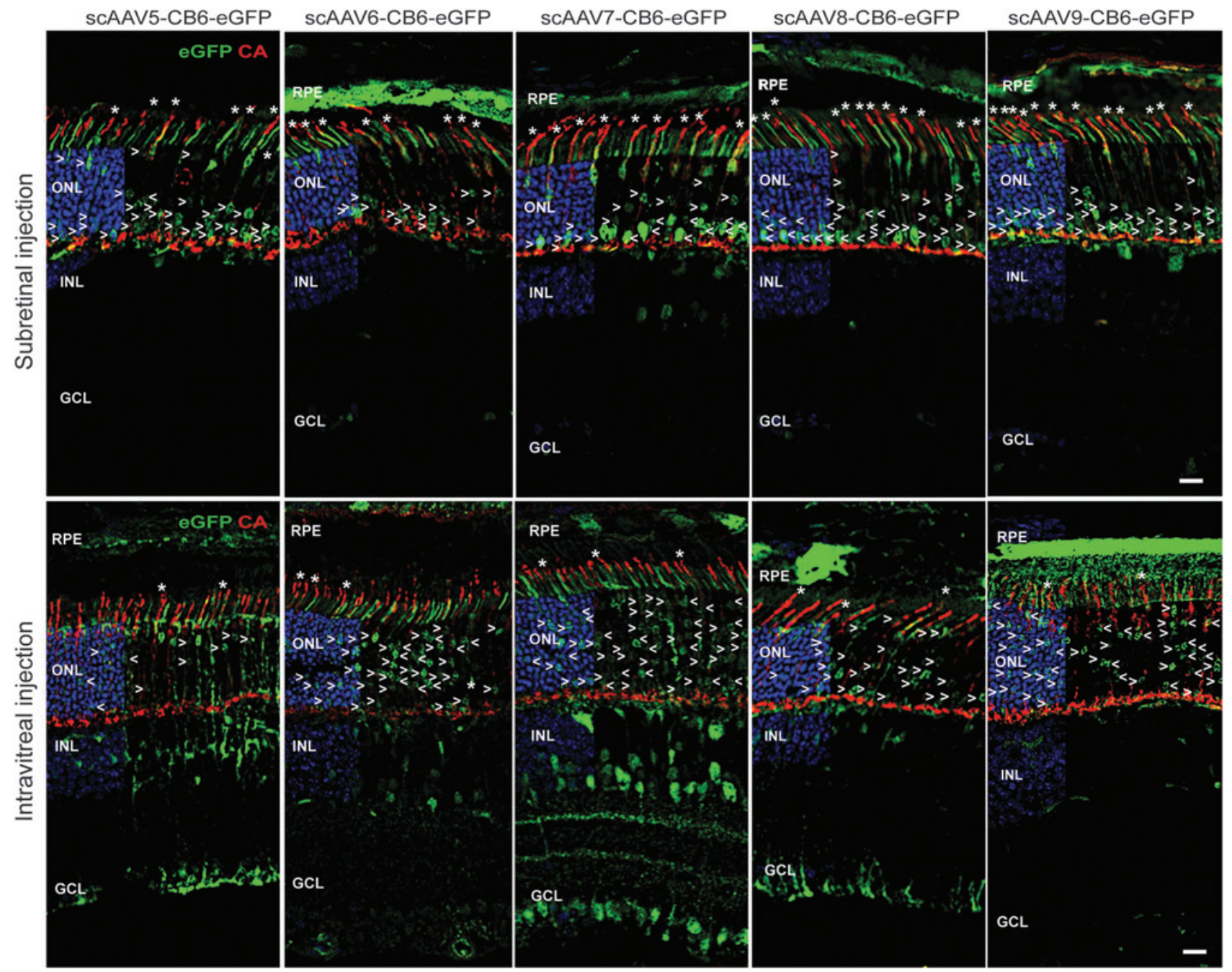

Figure 4. Comparative analysis of $A A V$ vector transduction in the mouse retina after subretinal or intravitreal injection at PND1. PND1 mice injected either subretinally (upper row) or intravitreally (lower row) with scAAV5, -6, -7, -8, or -9 expressing eGFP under the control of the CB6 promoter, as indicated. Histological analysis was performed at 3 wpi on cryosections stained with CA (red) and counterstained with DAPI (blue). AAV-mediated GFP was detected by its native fluorescence (green). Subretinal injections result in a higher number of transduced cones (asterisks) with most of the transduced rods (arrowheads) located in the inner most part of the ONL (see also Figs. 1 and 2), while intravitreal injections result in scattered rods throughout the 0NL and fewer transduced cones. Scale bars $=25 \mu \mathrm{m}$. GCL, ganglion cell layer.

ready post-mitotic by $\mathrm{PND} 1^{44}$ and lineage studies using retroviral vectors have revealed that most rod progenitor cells that persist in the rodent retina after birth are terminally dividing, ${ }^{49-51}$ rod progenitors may dilute or lose AAV genomes, resulting in consistent decreased transgene expression in mature rods. Alternatively or concomitantly, the overall absence of efficient rod transduction at PND1 may relate to the progressive transcriptional silencing of AAV vector DNA due to developmental epigenetic changes. Indeed, histone modifications have been shown to be important contributors to gene regulation in developing rod PRs. ${ }^{52}$

To investigate these two possibilities, AAV5, -6, $-7,-8$, and -9 were intravitreally injected in PND1 mice. If the factors that determine AAV tropism during PR development are related to AAV vector DNA, the final pattern of transgene expression should globally be similar between the two routes of administration. However, for all five serotypes, it was found that rods were the predominant PR cells transduced when the vectors were injected intravitreally (e.g., for AAV5: $4.2 \pm 2.6 \%$ of rods vs. $0.9 \pm 0.3 \%$ of cones near the injection site; $n=3$; Fig. 4 and Supplementary Fig. S7). Notably, rod nuclei expressing detectable levels of GFP were evenly distributed across the ONL after intravitreal injection (Fig. 4 and Supplementary Fig. S7), indicating that lack of efficient AAVmediated transduction of the late-born rods by subretinal injections at PND1 is not due to cell division or differential silencing of the vector ge- 
nomes. The data suggest that factors affecting the efficiency of AAV-mediated transduction of early and late-born rods are site specific and likely involve the access of AAV particles to the PR cells. Receptors necessary for binding/entry of AAVs while present in PRs may not yet be accessible on late-born cells by AAVs from the subretinal space.

\section{Lack of rod outer segments markedly alters PR tropism of AAV in the adult retina}

The timing of rod precursor birth (after E19) is tightly linked to the onset of PR segment formation and rhodopsin expression. ${ }^{53}$ To test whether the growth of rod segments and/or the associated matrix (Supplementary Fig. S8a and b) plays a role in the access of AAV particles to PRs, an attempt was made to visualize the distribution of AAV particles after subretinal injection at PND1 and PND21 using anti-AAV capsid antibodies. However, the large number of capsids present in the subretinal space limited the analysis (data not shown). Instead, qPCR was first employed to quantify viral binding on PND1 and PND21 retinas after modification of the rod IPM with neuraminidase (Supplementary Fig. S8). Ex vivo retinal explants were used to limit injection variability related to the surface of the retina exposed to the vector after subretinal delivery at PND1 versus PND21. We found that the rodassociated matrix could specifically interact with AAV particles in vitro (Supplementary Fig. S8d). However, these interactions were not conserved across AAV5, $-7,-8$, and -9 , indicating that binding of AAV to the rod IPM does not govern the common increase in rod transduction observed during PR development after subretinal delivery.

Next, to determine if the development of a normal rod segment plays a role in the access of AAV particles to PRs, rod and cone transduction was quantified in rhodopsin knockout $\left(R h o^{-/}\right)$mice ${ }^{54}$ after subretinal injection of AAV2/5-CMV-dGFP, AAV2/5-CMV-H2bGFP, and AAV2/5-pQCMV$H 2 b G F P$ vectors at PND1 and PND21 (Table 1 and Fig. 5). The $R \mathrm{Ro}^{-/-}$mouse is a well-characterized model of rod-cone dystrophy that does not develop rod outer segments. ${ }^{40}$ Importantly, this mouse model does not display any rod degeneration during the first three postnatal weeks. No alterations in the IPM and outer limiting membrane (OLM) ${ }^{55,56}$ were detected in $\mathrm{Rho}^{-/-}$mice at PND21 (Supplementary Fig. S9). In PND1-injected mice, the transduction profile was similar between $R h^{-/-}$ and wild-type mice, with a preferential transduction of cone PRs (Fig. 5a and c). However, PND21 injections resulted in a fairly dramatic difference in the final expression patterns. On average, the three
AAV vectors tested showed a similarly low transduction of rod PRs in $R h o^{-1-}$ retinas, as seen with PND1 injections in healthy retinas (Table 1 and Fig. 5a and c). Rod transduction was mainly observed at the site of injection near the needle tract (data not shown). $\mathrm{GFP}^{+}$rods were located in the outermost part of the ONL (Fig. 5a, arrows) and expressed qualitatively less GFP than cone PRs (Fig. 5a and b). These observations suggest that the presence of a sick rod segment can influence the efficiency rod transduction upon subretinal delivery in the adult retina. Furthermore, they suggest a potential correlation between altered rod transduction and increased cone transduction.

To delineate further the influence of rods on the efficiency of cone transduction, the $r d 1$ mouse model was utilized, in which the vast majority of rods are lost by PND21. ${ }^{57}$ However, in contrast to the $R \mathrm{Ro}^{-/-}$ retinas, the IPM is inevitably altered in this mouse model due to the loss of rods. AAV5 or AAV9 vectors were subretinally injected into PND21 $r d 1$ mice and cone transduction was evaluated on retinal flat mounts at 3 wpi (Table 1 and Fig. 5d). All vectors resulted in dramatically enhanced cone transduction compared to control mice, considering the percentage of cells transduced (Table 1). The consistent finding in two mouse models of rod degeneration that cone transduction increases in the absence of efficient rod transduction or in the absence of rods suggest that rods may negatively affect cone transduction in the adult mouse retina.

\section{DISCUSSION}

Gene therapy targeting PRs holds great potential for the treatment of many forms of retinal diseases, such as retinitis pigmentosa, Leber congenital amaurosis, or age-related macular degeneration. ${ }^{13} \mathrm{~A}$ common hallmark of these diseases is the progressive dysfunction, degeneration, and death of PR cells. The first sign of these diseasesvisual impairment-is generally associated with early alterations of the PRs functional structures, the inner and outer segments, while PR cell bodies are lost later in the process.

This study found that PR development and integrity play a major role in the efficacy of AAVmediated transduction after subretinal injection. It confirmed that rod transduction increases dramatically during mouse postnatal retinal development, ${ }^{30-34}$ irrespective of the AAV serotype (Fig. 1 and Supplementary Fig. S6), and showed that rod transduction correlates with the differentiation order of PR cells (Fig. 2 and Supplementary Figs. S4 and S5). This effect was not due to differ- 
a

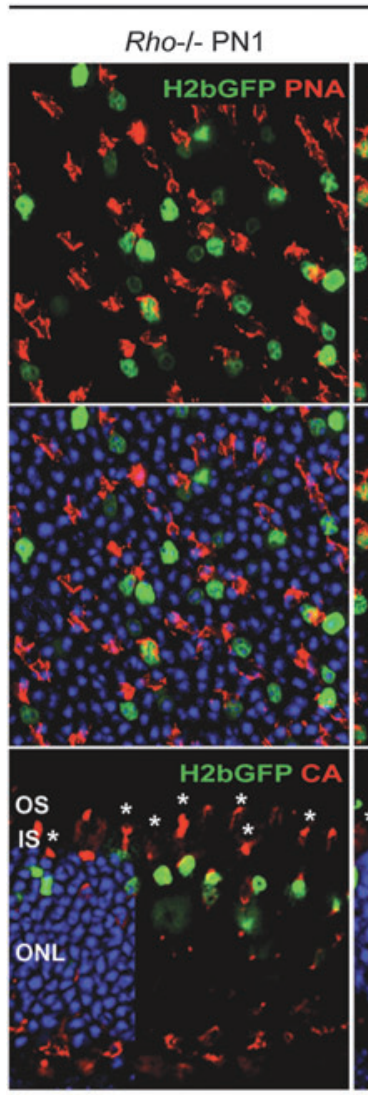

sSAAV5-CMV-H2bGFP

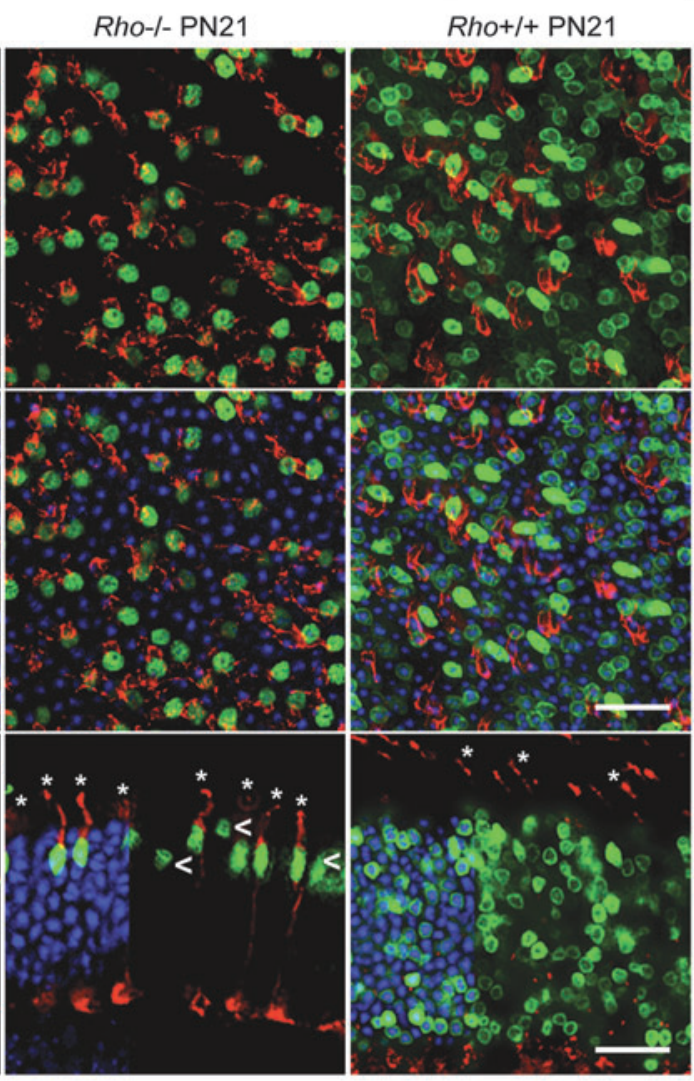

b

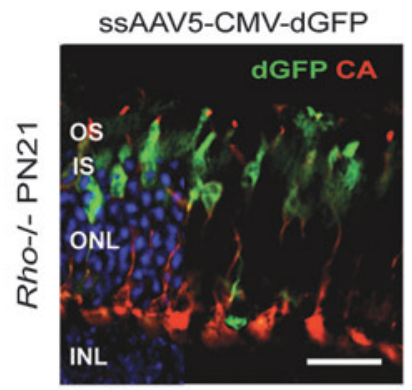

C

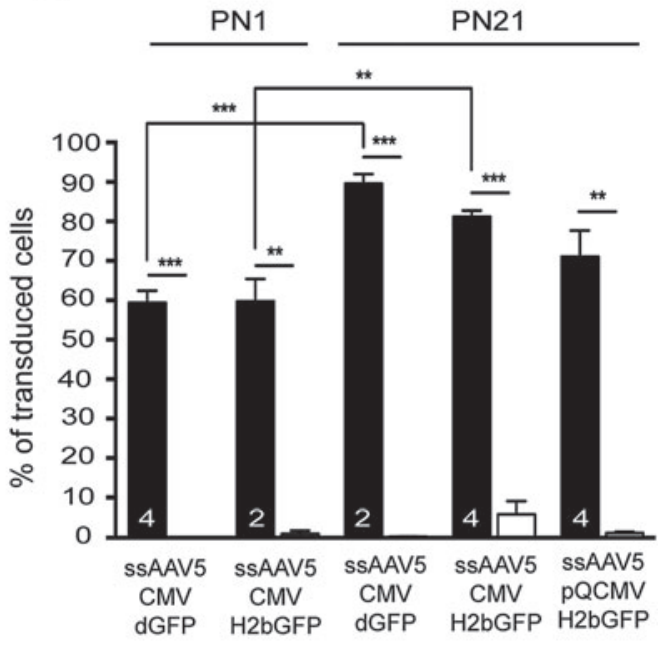

d

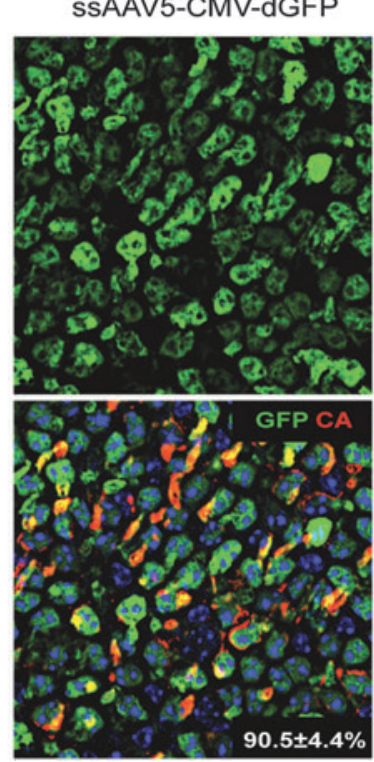

ssAAV5-CMV-H2bGFP

scAAV5-CB6-eGFP
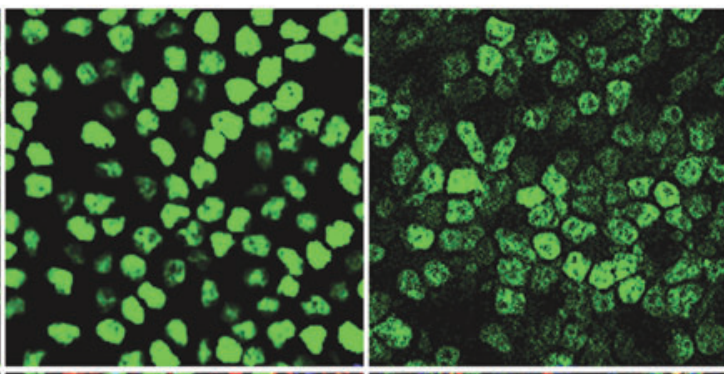

scAAV9-CB6-eGFP

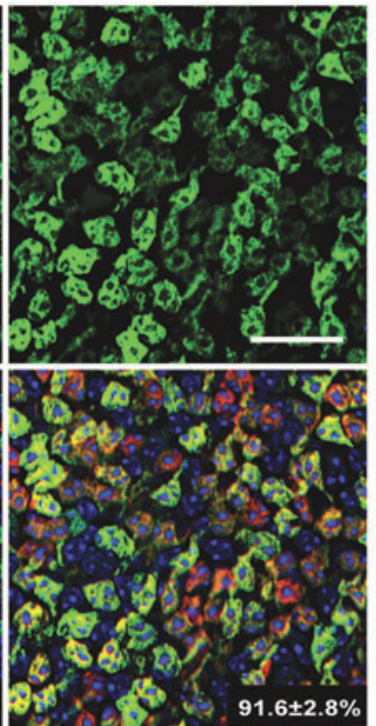

Figure 5. Altered of AAV tropism in Rho ${ }^{-1-}$ and adult $r d 1$ mice. (a-c) PND1 and PND21 Rho ${ }^{-/}$mice injected with similar doses of AAV2/5-CMV-H2bGFP, AAV2/5-CMV-dGFP, or AAV2/5-pQCMV-H2bGFP. Three weeks after injection, retinas were labeled with PNA or with an antibody raised against CA (red). AAVmediated GFP was detected by its native fluorescence (green). Representative images after injection of (a) AAV2/5-CMV-H2bGFP and (b) AAV2/5-CMV- $d G F P$ at age indicated. Asterisks indicate $\mathrm{GFP}^{+}$cones and arrowheads indicate $\mathrm{GFP}^{+}$rods. Quantitative analysis of the percentage of $\mathrm{GFP}^{+}$cones (black bars) and rods (white bars) is shown in (c). Error bars represent $S D$. Numbers in bars represent the number of retina analyzed. ${ }^{* *} p<0.01$ and ${ }^{* * *} p<0.001$ by Student's $t$-test. (d) Retinal flat mounts of $r d 1$ mice injected at PND21 with similar doses of AAV2/5-CMV- $d G F P, A A V 2 / 5-C M V-H 2 b G F P$, scAAV2/5-CB6-eGFP, or scAAV2/9CB6-eGFP and stained for CA expression (red) 3 wpi. AAV-mediated GFP was detected by its native fluorescence (green). Percentage of transduced cones for each vector is indicated in corresponding panel as mean $\pm S D$. Scale bars $=25 \mu \mathrm{m}$. 
ences in promoter activity or second-strand synthesis of the viral genome, which in principle could account for the enhanced rod transduction (Fig. 1 and Supplementary Fig. S2). Neither was this effect related to the gradual exit of rod progenitors from the cell cycle or to epigenetic/protein inhibition of the vector genomes during development, since both early and late-born cells were efficiently transduced at PND1 after intravitreal delivery of AAV vectors (Fig. 4). Thus, while serotypeindependent (Figs. 3 and 4, and Supplementary Figs. S4-S6), the effects of PR development on AAV transduction were, however, site specific (Fig. 4).

It is possible that restriction to AAV transduction in immature rods occurs after virion internalization due to inefficient viral trafficking. However, intracellular AAV particle trafficking has been shown to be influenced by the viral capsid sequence, ${ }^{58}$ which in this case seems not to be relevant with regards to the common shift in tropism between PND1 and PND21 subretinal injections. Alternatively, the enhanced transduction of rods during retinal development could be related to increased entry of AAVs into PRs. The OLM, in which the zonulae adherents pore size has been estimated to be between 30 and $36 \AA,{ }^{59}$ may impede the diffusion of AAV capsids ( $250 \AA$ ) to the ONL, allowing efficient access (and thus entry) of AAV particles mainly to PRs that have started to developed inner segments that protrude through the OLM (i.e., cones and early-born rods). ${ }^{33}$ In support of this model, ${ }^{33}$ the timing of rod precursor birth (after E19) is known to be tightly linked to the onset of PR segment formation and rhodopsin expression. ${ }^{53}$ Concomitantly, the development of rod outer segment may directly or indirectly correlate with increased-nonspecific-entry of AAV vectors into PRs. Indeed, a shift in the distribution of $\mathrm{GFP}^{+}$rod nuclei was observed when AAV5 vectors were delivered after PND10, which coincides with the elongation of rod outer segments for the majority of rods at this age (Fig. 2c). Moreover, rod transduction was profoundly inhibited in adult $R h o^{-1-}$ retinas (Fig. 5) that lack rod outer segment development but still display rod inner segments that protrude through the OLM. Of course, other factors, such as PR stress and degeneration, could affect the expression of AAV receptors, as well as inhibit the expression of the transgene in the diseased retina. However, there is more rational evidence from the observations to suggest that vector access to PR segments is one of the host factors governing PR transduction efficiency upon subretinal delivery, in particular since at PND21, $R h o^{-/}$retinas had no apparent loss of rod PRs or alterations of the IPM and OLM integrity (Supplementary Fig. S9).

Importantly, the idea that PR outer segments modulate rod transduction in the adult retina does not contradict previous reports that demonstrated unequivocal (but transient) structural and/or functional rescue of rods by AAV-mediated gene replacement therapy in two mouse models of retinitis pigmentosa that fail to develop rod outer segments; namely the $R h o^{-1-16,60}$ and the $P h r p 2^{--61,62}$ mice, In the $R \mathrm{ho}^{-/-}$mice, therapeutic vectors were injected into neonatal mice and mice younger than PND5- two time points that precede rod outer segment growth in wild-type mice and where it has been shown that rod transduction is dependent on the viral load injected (Fig. 1d). In the Phrp $2^{-/-}$mice, it has been notably shown that there was a significant difference in the number and quality of outer segment rescue in treated retinas. Depending on the age at which the mice were treated, fewer outer segments developed normally, with the best results obtained in younger animals, despite the relatively slow rate of $\mathrm{PR}$ death in that model. Moreover, particular differences in the infectivity of the vector preparations and the presumed vector titers may exist between all these studies, making it difficult to cross-compare them directly. The goal of this study was to determine host cell factors that influence PR transduction, and oversaturating the system with AAV vectors would have been counterproductive in this case. As mentioned, it has previously been shown in wild-type mice that the majority of immature rods in addition to inner nuclear layer cells can be transduced by subretinal injection at PND1 if a higher dose of vector is used (Fig. 1d). ${ }^{41} \mathrm{In}$ this study, the rate of rod transduction after injection at PND21 was limited to 30-55\%, while other groups have reported PR transduction rates of $>90 \%$ within the vector exposed area. ${ }^{14,17}$ Thus, the overall lower transduction in this study can easily explain why in $R h o^{-1}$ mice such a large difference with PND21 subretinal injections was seen. Another factor that complicates the comparison between experiments in the $R h o^{-1-}$ mice and the previously published results by Palfi et al. ${ }^{16,54,60}$ is that the present study used different $R h o^{-/-}$mice. ${ }^{40}$ Strain background differences between the two strains and the way both knockout strains were generated could have exacerbated the effect of the lower transduction rate used to perform this study. In this regard, it is interesting to note that the group of Jean Bennett has also reported difficulties in transducing efficiently rods in the same $R h \mathrm{O}^{-/-}$model that was used in this study ${ }^{63}$ (Dejneka NS et al., ARVO Annual Meeting Abstract, 2002). 
The present findings provide important information regarding the effects of subretinal gene therapy in animal models of PR dystrophies. First, the results indicate that the time of intervention affects the efficacy of AAV transduction if gene therapy is applied before the full development of the retina. However, in several mouse models of PR dystrophies, the degeneration of PRs is so fast that most gene therapy attempts have been conducted during the first week of life ${ }^{13}$ due to the relative slow onset of AAV-mediated transgene expression. ${ }^{28}$ For instance, in the $n m f 363$ murine model of PDE6 $\alpha$-deficiency, no apparent PR degeneration is observed at PND12, but by PND14, $30 \%$ of PRs are already lost and only one row of PR nuclei remains in the ONL by PND38. ${ }^{64}$ In this model, injection of an AAV8(Y788F)-RHO-mPde6a at PND5 resulted in an initial loss of cells between 1 and 2 months of age, followed by a preservation of three to four rows of PR nuclei in the vector-exposed area, for at least 6 months. ${ }^{65}$ However, the overall rod functional rescue was too low to make a detectable difference by electroretinography. ${ }^{65}$ Notably, mid-stage intervention at PND21 achieved similar efficacy as PND5 treatment, despite loss of approximately half of PRs at the time of injection, ${ }^{66}$ indicating that the efficacy of PND5 treatment may have been limited by the efficient transduction of rods when compared to the PND21 intervention. As well, another study reported that subretinal injection of AAV2/5-smCBA$\mathrm{m} P$ de $6 \beta$ vector $\left(1 \times 10^{10}\right.$ genome copies $)$ in $r d 10$ mice at PND4 (before the onset of PR dystrophy) or PND21 (after the onset of PR dystrophy) resulted in similar (partial) therapeutic effects. ${ }^{67}$

Second, the results indicate that if $\mathrm{PR}$ integrity determines the ability of AAV vectors to target the $\mathrm{PRs}$, the spatio-temporal kinetics of retinal degeneration, the site of injection, and the health status of the retina at the time of treatment are also important factors affecting the overall efficacy of transduction. For instance, murine ${ }^{64,68-73}$ and canine ${ }^{74-77}$ models of severe PR dystrophies show significant loss of PR segments at early stages of the disease. In these models, most preclinical studies have demonstrated improved PR survival only when gene therapy is applied before or at very early stages of retinal degeneration. ${ }^{13,23,78-84}$ Moreover, when gene therapy has been applied at later stages of the disease, the proportion of PRs that were not responding to the treatment ("silent" cells) increased within the vectorexposed area. ${ }^{1,23,85}$ It has been first suggested that there is a threshold of accumulated changes after which $\mathrm{PR}$ death is inevitable. ${ }^{1}$ However, recent data indicate that continued PR loss may instead reflect insufficient transduction efficiencies. ${ }^{29}$ An intriguing possibility is that AAV vectors might not efficiently access and infect subpopulations of deteriorated PRs. Thus, early alterations of PRs may be associated with a higher heterogeneity of transduction within the vector-exposed area (e.g., $40 \%$ of PRs expressing high levels of transgene and $60 \%$ of PRs expressing low levels of transgenes rather than $100 \%$ of PRs expressing medium levels of transgene). An important step in approaching this problem will be to understand the longitudinal changes that occur during phases of PR stress/degeneration at the level of both the PRs and AAV vector pharmacology. Future studies will also have to be designed to determine whether a temporal disruption of the $\mathrm{OLM}^{86}$ and higher doses of vectors may be able to overcome this hurdle. Finally, delineating the relationship between PR development, AAV trafficking, and cell receptor usage will be essential toward developing a complete understanding of PR transduction upon subretinal injection.

It did not escape the authors' attention that the results support the notion that rods and cones may directly or indirectly compete for AAV access. Preclinical studies with several AAV serotypes have established that subretinal injection in adults often results in dominant transduction of rods within rod-rich retinas. ${ }^{15,19-22,42,87}$ For instance, in wild-type mice (cone:rod ratio of 1:30), while fairly high transduction of rods is observed after subretinal injection of $\mathrm{AAV} 2 / 8$, cone transduction is restricted to only $1-12 \%$ of cells. ${ }^{15}$ In pigs, AAV2/8 mediates 3.8- to 5- and 1.7-fold higher levels of PR transduction than AAV2/5 and AAV2/9, respectively. ${ }^{15,22}$ However, all serotypes transduce the same percentage of cones in the cone-enriched visual streak (cone:rod ratio of 1:3 to 1:5), with up to $9.2 \%$ of cones readily transduced. ${ }^{15}$ In dogs, AAV2/5 also preferentially targets rods, ${ }^{42}$ though significant cone transduction is observed in cone-enriched areas, ${ }^{23}$ with the use of high doses of vectors. ${ }^{43}$ Primate studies with seven different AAV serotypes reported similar findings with weak cone transduction in the rod-rich parafoveal region (1-10\%), even in the presence of ample rod PR transduction, and with higher levels of cone transduction (20 $50 \%$ ) in the pure-cone fovea. ${ }^{19-21,87}$

Consistent with the notion that there is a developmental effect in which access by AAV to cone PRs becomes restricted, a profound decrease in cone transduction was observed in the developing mouse retina (Fig. 2). These observations appear to correlate with the increase in rod transduction (Fig. 2b). Moreover, cone transduction was significantly enhanced after subretinal injection of AAV vectors in PND21 Rho ${ }^{-1-}$ (Fig. 5a-c) and $r d 1$ mice (Fig. 5d), in which rod transduction was inhibited by the absence 
of a healthy rod segments or by the loss of the vast majority of rod cells at the time of treatment. Although comprehensive analysis will be required to elucidate the details of PR transduction upon subretinal injection, it should be noted that there is no evidence from the present observations to suggest that AAV particles use different receptors between rods and cones. Indeed, a similar shift in tropism was observed for all tested AAV serotypes (Fig. 3 and Supplementary Fig. S6). It is possible that the densely stacked rod outer segments form physical barriers between the site of delivery and the cones. Concomitantly, cone matrix sheaths located around cone inner and outer segments may form a barrier that selectively reduces access of AAV particles to the cones. Interestingly, a recent study in the feline retina has shown that unlike mice and nonhuman primates, cone PRs were more efficiently transduced than rods. ${ }^{26}$ The reasons for this difference in cellular tropism remain unknown, but the cone matrix sheath of the cat differs significantly from other mammalian species. ${ }^{88}$ In primates, only AAV9 was shown to target cones both centrally and peripherally efficiently at low doses when directly compared to five other AAV serotypes. ${ }^{20}$ It has been hypothesized that this property is due to the abundance of terminal galactose, the cell receptor for AAV9 on the cone PR matrix. ${ }^{20}$

The notion that rods and cones compete for AAV transduction emphasizes the need of evaluating components of $\mathrm{AAV}$ vectors planned for humans in models that accurately depict physiological characteristics of the human retina (i.e., large animals and all-cone murine retinas). ${ }^{15,20-23,42,43,89,90}$ Indeed, while in humans macular cones will often be the primary treatment area, the ability of AAV vectors to transduce the cones and restore their function is often evaluated in murine models of $\mathrm{PR}$ dystrophies that do not display a cone-enriched area but a high rod:cone ratio. It also indicates that loss of rod PRs associated with many forms of retinopathies could be exploited to redirect AAVs commonly toward cones.

\section{ACKNOWLEDGMENTS}

We are grateful to Christian Mueller (UMASS Medical School) for insightful discussions and critical reading of the manuscript; to Qin $\mathrm{Su}$ (UMASS Medical School) and the Vector Core of the Horae Gene Therapy Center for the production of the scAAV-CB6-eGFP vectors; to Sourav Choudhury and Miguel Sena-Esteves (UMASS Medical School) for the gift of the Pro5 and Lec2 cell lines; and to Julio Sanmiguel (UMASS Medical School) for the gift of the eGFP qPCR primers. This work was supported by an unrestricted grant from "Information Recherche Retinite Pigmentaire" Association (L.P.) and by the US National Institutes of Health (RO1-EY023570, C.P). L.P. also acknowledges the following funding: the Fulbright/ Fondation Monahan Postdoctoral Fellowship, the Fondation de France "Young researcher in ophthalmology" Fellowship, and the Association Française contre les Myopathies (AFM-Telethon) Postdoctoral Fellowship.

\section{AUTHOR DISCLOSURE}

G.G. is a founder of Voyager Therapeutics, specialized in AAV-based gene therapy for the central nervous system, and holds equity in the company. G.G. is an inventor of patents with potential royalties licensed to Voyager Therapeutics and other biopharmaceutical companies. No competing financial interests exist for the remaining authors.

\section{REFERENCES}

1. Cideciyan AV, Jacobson SG, Beltran WA, et al. Human retinal gene therapy for Leber congenital amaurosis shows advancing retinal degeneration despite enduring visual improvement. Proc Natl Acad Sci U S A 2013;110:E517-525.

2. Bainbridge JW, Mehat MS, Sundaram V, et al. Long-term effect of gene therapy on Leber's congenital amaurosis. N Engl J Med 2015;372: 1887-1897.

3. Bainbridge JW, Smith AJ, Barker SS, et al. Effect of gene therapy on visual function in Leber's congenital amaurosis. N Engl J Med 2008;358: 2231-2239.
4. Bennett J, Ashtari M, Wellman J, et al. AAV2 gene therapy readministration in three adults with congenital blindness. Sci Transl Med 2012;4: 120ra115

5. Bennett J, Wellman J, Marshall KA, et al. Safety and durability of effect of contralateral-eye administration of AAV2 gene therapy in patients with childhood-onset blindness caused by RPE65 mutations: a follow-on Phase 1 trial. Lancet 2016; 388:661-672.

6. Maguire AM, Simonelli F, Pierce EA, et al. Safety and efficacy of gene transfer for Leber's con- genital amaurosis. N Engl J Med 2008;358:22402248.

7. Hauswirth WW, Aleman TS, Kaushal S, et al. Treatment of leber congenital amaurosis due to RPE65 mutations by ocular subretinal injection of adeno-associated virus gene vector: short-term results of a Phase I trial. Hum Gene Ther 2008; 19:979-990.

8. Jacobson SG, Cideciyan AV, Roman AJ, et al. Improvement and decline in vision with gene therapy in childhood blindness. N Engl J Med 2015; 372:1920-1926. 
9. Weleber RG, Pennesi ME, Wilson DJ, et al. Results at 2 years after gene therapy for RPE65deficient Leber congenital amaurosis and severe early-childhood-onset retinal dystrophy. Ophthalmology 2016;123:1606-1620.

10. Ghazi NG, Abboud EB, Nowilaty SR, et al. Treatment of retinitis pigmentosa due to MERTK mutations by ocular subretinal injection of adenoassociated virus gene vector: results of a Phase I trial. Hum Genet 2016;135:327-343.

11. Edwards TL, Jolly JK, Groppe M, et al. Visual acuity after retinal gene therapy for choroideremia. N Engl J Med 2016;374:1996-1998.

12. MacLaren RE, Groppe M, Barnard AR, et al. Retinal gene therapy in patients with choroideremia: initial findings from a Phase 1/2 clinical trial. Lancet 2014;383:1129-1137.

13. Petit L, Khanna $H$, Punzo C. Advances in gene therapy for diseases of the eye. Hum Gene Ther 2016;27:563-579.

14. Allocca M, Mussolino C, Garcia-Hoyos M, et al Novel adeno-associated virus serotypes efficiently transduce murine photoreceptors. J Virol 2007;81: $11372-11380$

15. Manfredi A, Marrocco E, Puppo A, et al. Combined rod and cone transduction by adeno-associated virus 2/8. Hum Gene Ther 2013;24:982-992.

16. Palfi A, Chadderton N, O'Reilly M, et al. Efficient gene delivery to photoreceptors using AAV2/rh10 and rescue of the Rho(-l-) mouse. Mol Ther Methods Clin Dev 2015;2:15016.

17. Natkunarajah M, Trittibach P, Mclntosh J, et al. Assessment of ocular transduction using singlestranded and self-complementary recombinant adeno-associated virus serotype 2/8. Gene Ther 2008;15:463-467.

18. Auricchio A, Kobinger G, Anand V, et al. Exchange of surface proteins impacts on viral vector cellular specificity and transduction characteristics: the retina as a model. Hum Mol Genet 2001;10:30753081.

19. Vandenberghe LH, Bell P, Maguire AM, et al Dosage thresholds for AAV2 and AAV8 photoreceptor gene therapy in monkey. Sci Transl Med 2011;3:88ra54.

20. Vandenberghe $L H$, Bell $P$, Maguire $A M$, et al. AAV9 targets cone photoreceptors in the nonhuman primate retina. PLoS One 2013;8:e53463.

21. Boye SE, Alexander JJ, Boye SL, et al. The human rhodopsin kinase promoter in an AAV5 vector confers rod- and cone-specific expression in the primate retina. Hum Gene Ther 2012;23:11011115.

22. Mussolino C, della Corte M, Rossi S, et al. AAVmediated photoreceptor transduction of the pig cone-enriched retina. Gene Ther 2011;18:637645.

23. Lheriteau E, Petit L, Weber M, et al. Successful gene therapy in the RPGRIP1-deficient dog: a large model of cone-rod dystrophy. Mol Ther 2014;22:265-277.
24. Bruewer AR, Mowat FM, Bartoe JT, et al. Evaluation of lateral spread of transgene expression following subretinal AAV-mediated gene delivery in dogs. PLoS One 2013;8:e60218.

25. Stieger K, Colle MA, Dubreil L, et al. Subretinal delivery of recombinant AAV serotype 8 vector in dogs results in gene transfer to neurons in the brain. Mol Ther 2008;16:916-923.

26. Minella AL, Mowat FM, Willett KL, et al. Differential targeting of feline photoreceptors by recombinant adeno-associated viral vectors: implications for preclinical gene therapy trials. Gene Ther 2014; 21:913-920.

27. Surace EM, Auricchio A. Versatility of AAV vectors for retinal gene transfer. Vision Res 2008;48:353359 .

28. Vandenberghe LH, Auricchio A. Novel adenoassociated viral vectors for retinal gene therapy. Gene Ther 2012;19:162-168.

29. Koch SF, Tsai YT, Duong JK, et al. Halting progressive neurodegeneration in advanced retinitis pigmentosa. J Clin Invest 2015;125:3704-3713.

30. Surace EM, Auricchio A, Reich SJ, et al. Delivery of adeno-associated virus vectors to the fetal retina: impact of viral capsid proteins on retinal neuronal progenitor transduction. J Virol 2003;77: 7957-7963

31. Pang JJ, Lauramore A, Deng WT, et al. Comparative analysis of in vivo and in vitro AAV vector transduction in the neonatal mouse retina: effects of serotype and site of administration. Vision Res 2008:48:377-385.

32. Watanabe S, Sanuki R, Ueno S, et al. Tropisms of AAV for subretinal delivery to the neonatal mouse retina and its application for in vivo rescue of developmental photoreceptor disorders. PLoS One 2013;8:e54146

33. Xiong W, Cepko C. Distinct expression patterns of AAV8 vectors with broadly active promoters from subretinal injections of neonatal mouse eyes at two different ages. Adv Exp Med Biol 2016;854: 501-507

34. Xiong W, MacColl Garfinkel AE, Li Y, et al. NRF2 promotes neuronal survival in neurodegeneration and acute nerve damage. J Clin Invest 2015;125: 1433-1445.

35. Punzo C, Cepko CL. Ultrasound-guided in utero injections allow studies of the development and function of the eye. Dev Dyn 2008;237:10341042.

36. Rashnonejad A, Chermahini GA, Li S, et al. Largescale production of adeno-associated viral vector serotype-9 carrying the human survival motor neuron gene. Mol Biotechnol 2016;58:30-36.

37. Grieger JC, Choi VW, Samulski RJ. Production and characterization of adeno-associated viral vectors Nat Protoc 2006:1:1412-1428.

38. Ayuso E, Mingozzi F, Montane J, et al. High AAV vector purity results in serotype- and tissueindependent enhancement of transduction efficiency. Gene Ther 2010;17:503-510.
39. Le YZ, Ash JD, Al-Ubaidi MR, et al. Targeted expression of Cre recombinase to cone photoreceptors in transgenic mice. Mol Vis 2004;10: 1011-1018

40. Lem J, Krasnoperova NV, Calvert PD, et al. Morphological, physiological, and biochemical changes in rhodopsin knockout mice. Proc Natl Acad Sci U S A 1999;96:736-741.

41. Venkatesh $A$, Ma $S$, Langellotto $F$, et al. Retinal gene delivery by rAAV and DNA electroporation. Curr Protoc Microbiol 2013;Chapter 14:Unit 14D 14.

42. Beltran WA, Boye SL, Boye SE, et al. rAAV2/5 gene-targeting to rods:dose-dependent efficiency and complications associated with different promoters. Gene Ther 2010;17:1162-1174.

43. Komaromy AM, Alexander JJ, Cooper AE, et al Targeting gene expression to cones with human cone opsin promoters in recombinant AAV. Gene Ther 2008:15:1049-1055.

44. Swaroop A, Kim D, Forrest D. Transcriptional regulation of photoreceptor development and homeostasis in the mammalian retina. Nat Rev Neurosci 2010;11:563-576.

45. Mack AF, Papanikolaou D, Lillo C. Investigation of the migration path for new rod photoreceptors in the adult cichlid fish retina. Exp Neurol 2003;184: 90-96.

46. Mack AF, Fernald RD. New rods move before differentiating in adult teleost retina. Dev Biol 1995:170:136-141.

47. Henderson RG, Fernald RD. Timing and location of rhodopsin expression in newly born rod photoreceptors in the adult teleost retina. Brain Res Dev Brain Res 2004;151:193-197.

48. Fei Y. Development of the cone photoreceptor mosaic in the mouse retina revealed by fluorescent cones in transgenic mice. Mol Vis 2003;9: 31-42.

49. Cepko C. Intrinsically different retinal progenitor cells produce specific types of progeny. Nat Rev Neurosci 2014;15:615-627.

50. Turner DL, Cepko CL. A common progenitor for neurons and glia persists in rat retina late in development. Nature 1987;328:131-136.

51. Hafler BP, Surzenko N, Beier KT, et al. Transcription factor Olig2 defines subpopulations of retinal progenitor cells biased toward specific cell fates. Proc Natl Acad Sci U S A 2012;109:78827887

52. Mo A, Luo C, Davis FP, et al. Epigenomic landscapes of retinal rods and cones. Elife 2016; 5:e11613

53. Morrow EM, Belliveau MJ, Cepko CL. Two phases of rod photoreceptor differentiation during rat retinal development. J Neurosci 1998;18:37383748

54. Humphries MM, Rancourt D, Farrar GJ, et al Retinopathy induced in mice by targeted disruption of the rhodopsin gene. Nat Genet 1997;15 216-219. 
55. Jaissle GB, May CA, Reinhard J, et al. Evaluation of the rhodopsin knockout mouse as a model of pure cone function. Invest Ophthalmol Vis Sci 2001:42:506-513.

56. Calame M, Cachafeiro M, Philippe $S$, et al. Retinal degeneration progression changes lentiviral vector cell targeting in the retina. PLoS One 2011; 6:e23782.

57. Punzo C, Kornacker K, Cepko CL. Stimulation of the insulin/mTOR pathway delays cone death in a mouse model of retinitis pigmentosa. Nat Neurosci 2009;12:44-52.

58. Johnson JS, Li C, DiPrimio N, et al. Mutagenesis of adeno-associated virus type 2 capsid protein VP1 uncovers new roles for basic amino acids in trafficking and cell-specific transduction. J Virol 2010;84:8888-8902.

59. Bunt-Milam AH, Saari JC, Klock IB, et al. Zonulae adherentes pore size in the external limiting membrane of the rabbit retina. Invest Ophthalmol Vis Sci 1985;26:1377-1380.

60. Palfi A, Millington-Ward S, Chadderton N, et al. Adeno-associated virus-mediated rhodopsin replacement provides therapeutic benefit in mice with a targeted disruption of the rhodopsin gene. Hum Gene Ther 2010;21:311-323.

61. Ali RR, Sarra GM, Stephens C, et al. Restoration of photoreceptor ultrastructure and function in retinal degeneration slow mice by gene therapy. Nat Genet 2000;25:306-310.

62. Sarra GM, Stephens C, de Alwis M, et al. Gene replacement therapy in the retinal degeneration slow (rds) mouse: the effect on retinal degeneration following partial transduction of the retina. Hum Mol Genet 2001;10:2353-2361.

63. Liang FQ, Dejneka NS, Cohen DR, et al. AAVmediated delivery of ciliary neurotrophic factor prolongs photoreceptor survival in the rhodopsin knockout mouse. Mol Ther 2001;3:241-248.

64. Sakamoto K, McCluskey M, Wensel TG, et al. New mouse models for recessive retinitis pigmentosa caused by mutations in the Pde6a gene. Hum Mol Genet 2009;18:178-192.

65. Wert KJ, Davis RJ, Sancho-Pelluz J, et al. Gene therapy provides long-term visual function in a pre-clinical model of retinitis pigmentosa. Hum Mol Genet 2013;22:558-567.

66. Wert KJ, Sancho-Pelluz J, Tsang SH. Mid-stage intervention achieves similar efficacy as conventional early-stage treatment using gene therapy in a pre-clinical model of retinitis pigmentosa. Hum Mol Genet 2014;23:514-523.
67. Yao J, Jia L, Khan N, et al. Caspase inhibition with XIAP as an adjunct to AAV vector genereplacement therapy: improving efficacy and prolonging the treatment window. PLoS One 2012; 7:e37197.

68. Blanks JC, Adinolfi AM, Lolley RN. Photoreceptor degeneration and synaptogenesis in retinal-degenerative (rd) mice. J Comp Neurol 1974;156:95-106.

69. LaVail MM, Sidman RL. C57BL-6J mice with inherited retinal degeneration. Arch Ophthalmol 1974;91:394-400.

70. Tansley K. Hereditary degeneration of the mouse retina. Br J Ophthalmol 1951;35:573-582.

71. Gargini C, Terzibasi E, Mazzoni F, et al. Retinal organization in the retinal degeneration $10(r d 10)$ mutant mouse: a morphological and ERG study. J Comp Neurol 2007;500:222-238.

72. Won J, Gifford E, Smith RS, et al. RPGRIP1 is essential for normal rod photoreceptor outer segment elaboration and morphogenesis. Hum Mol Genet 2009;18:4329-4339.

73. Ramamurthy V, Niemi GA, Reh TA, et al. Leber congenital amaurosis linked to AIPL1: a mouse model reveals destabilization of cGMP phosphodiesterase. Proc Natl Acad Sci U S A 2004; 101:13897-13902.

74. Parry HB. Degenerations of the dog retina. II. Generalized progressive atrophy of hereditary origin. Br J Ophthalmol 1953;37:487-502.

75. Aguirre GD, Rubin LF. Rod-cone dysplasia (progressive retinal atrophy) in Irish setters. J Am Vet Med Assoc 1975;166:157-164.

76. Tuntivanich N, Pittler SJ, Fischer AJ, et al. Characterization of a canine model of autosomal recessive retinitis pigmentosa due to a PDE6A mutation. Invest Ophthalmol Vis Sci 2009;50:801-813.

77. Beltran WA, Acland GM, Aguirre GD. Agedependent disease expression determines remodeling of the retinal mosaic in carriers of RPGR exon ORF15 mutations. Invest Ophthalmol Vis Sci 2009;50:3985-3995.

78. Petit L, Lheriteau E, Weber M, et al. Restoration of vision in the pde6beta-deficient dog, a large animal model of rod-cone dystrophy. Mol Ther 2012;20:2019-2030.

79. Pang JJ, Boye SL, Kumar A, et al. AAV-mediated gene therapy for retinal degeneration in the rd10 mouse containing a recessive PDEbeta mutation. Invest Ophthalmol Vis Sci 2008;49:4278-4283.

80. Pang JJ, Dai $X$, Boye SE, et al. Long-term retinal function and structure rescue using capsid mutant AAV8 vector in the rd10 mouse, a model of re- cessive retinitis pigmentosa. Mol Ther 2011;19: 234-242.

81. Sun X, Pawlyk B, Xu X, et al. Gene therapy with a promoter targeting both rods and cones rescues retinal degeneration caused by AIPL1 mutations. Gene Ther 2010;17:117-131.

82. Ku CA, Chiodo VA, Boye SL, et al. Gene therapy using self-complementary Y733F capsid mutant AAV2/8 restores vision in a model of early onset Leber congenital amaurosis. Hum Mol Genet 2011; 20:4569-4581.

83. Pawlyk BS, Smith AJ, Buch PK, et al. Gene replacement therapy rescues photoreceptor degeneration in a murine model of Leber congenital amaurosis lacking RPGRIP. Invest Ophthalmol Vis Sci 2005;46:3039-3045.

84. Koch S, Sothilingam V, Garcia Garrido M, et al. Gene therapy restores vision and delays degeneration in the CNGB1(-/-) mouse model of retinitis pigmentosa. Hum Mol Genet 2012;21:4486-4496.

85. Beltran WA, Cideciyan AV, Iwabe $S$, et al. Successful arrest of photoreceptor and vision loss expands the therapeutic window of retinal gene therapy to later stages of disease. Proc Natl Acad Sci U S A 2015;112:E5844-5853.

86. West EL, Pearson RA, Tschernutter M, et al. Pharmacological disruption of the outer limiting membrane leads to increased retinal integration of transplanted photoreceptor precursors. Exp Eye Res 2008;86:601-611.

87. Bennett J, Maguire AM, Cideciyan AV, et al. Stable transgene expression in rod photoreceptors after recombinant adeno-associated virus-mediated gene transfer to monkey retina. Proc Natl Acad Sci U S A 1999:96:9920-9925.

88. Fariss RN, Anderson DH, Fisher SK. Comparison of photoreceptor-specific matrix domains in the cat and monkey retinas. Exp Eye Res 1990;51:473-485.

89. Boye SL, Peterson JJ, Choudhury S, et al. Gene therapy fully restores vision to the all-cone Nrl(-/-) Gucy2e(-/-) mouse model of Leber congenital amaurosis-1. Hum Gene Ther 2015;26: 575-592.

90. Ye GJ, Budzynski E, Sonnentag P, et al. Conespecific promoters for gene therapy of achromatopsia and other retinal diseases. Hum Gene Ther 2016;27:72-82.

Received for publication January 24, 2017; accepted after revision May 16, 2017.

Published online: May 16, 2017. 